The AstrophysiCal Journal, 364:471-495, 1990 December 1

(C) 1990. The American Astronomical Society. All rights reserved. Printed in U.S.A.

\title{
THE OPTICAL EMISSION-LINE NEBULAE OF POWERFUL FAR-INFRARED GALAXIES
}

\author{
LeE Armus ${ }^{1}$ AND Timothy M. HeCKMan ${ }^{1}$ \\ Department of Physics and Astronomy, The Johns Hopkins University \\ AND \\ George K. Miley ${ }^{1}$ \\ Leiden Observatory; and Space Telescope Science Institute \\ Received 1990 January 26; accepted 1990 May 30
}

\begin{abstract}
We present and analyze narrow-band $(\mathrm{H} \alpha+[\mathrm{N} \mathrm{II}])$ emission-line images of a sample of 30 powerful farinfrared galaxies (FIRGs) chosen to have far-infrared spectral energy distributions similar to the prototype FIRGs Arp 220, NGC 3690, NGC 6240, and M82. We also present similar images of 10 other powerful farinfrared galaxies not chosen with regard to IR spectral shape. The emission-line nebulae of these IR colorselected (ICSS) FIRGs as a class are impressively large, with a mean half-light radius, $r_{e} \sim 1.3 \mathrm{kpc}$, and mean maximum diameter, $D \sim 16 \mathrm{kpc}$. They have morphologies that range from smooth and structureless to highly complex and chaotic, with some showing evidence for ordered structure on scales of a few $\mathrm{kpc}$ to tens of kpc. Only $\sim 25 \%$ of the nearby $(z \leq 0.10)$ FIRGs for which we have adequate spatial resolution display morphologies suggesting that large $\mathrm{H}$ II regions contribute significantly to their appearance in $\mathrm{H} \alpha+[\mathrm{N}$ II $]$. Of the seven ICSS FIRGs that are apparently highly flattened in $R$ (major-to-minor axis ratio $\geq 2.0$ ), six have

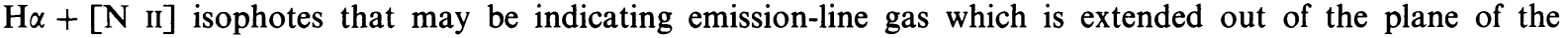
galaxy. This type of structure is also seen in three edge-on galaxies that are not members of the IR colorselected sample.

The mean total $\mathrm{H} \alpha+[\mathrm{N}$ II $]$ luminosity (uncorrected for internal extinction) of the ICSS FIRG nebulae is $\sim 5 \times 10^{41}$ ergs $\mathrm{s}^{-1}$, which is a factor of $2-3$ greater than that found for individual members of pairs of optically selected interacting galaxies (Bushouse, Lamb, and Werner), and a factor of $\sim 5$ greater than that of isolated spiral galaxies (Kennicutt and Kent). The eight sample galaxies with available $\mathrm{Br} \alpha / \mathrm{H} \alpha$ flux ratios have typical extinctions of $\sim 3 \mathrm{mag}$ at $\mathrm{H} \alpha$. This extinction then implies an intrinsic $\mathrm{H} \alpha+[\mathrm{N}$ II $]$ luminosity of $\sim 8 \times 10^{42}$ ergs s $^{-1}$ for the ICSS FIRGs, which is a factor of $\sim 30$ greater than the average extinction corrected $\mathrm{H} \alpha+[\mathrm{N}$ II] luminosity of isolated spiral galaxies of Kennicutt and Kent. The ICSS FIRGs are clearly extraordinary objects in terms of their optical line emission.

The good correlation of both $\mathrm{H} \alpha+[\mathrm{N} \mathrm{II}]$ and far-infrared luminosity with absolute magnitude, along with previous spectroscopic evidence that the optical continuum is produced predominantly by young stars (Armus, Heckman, and Miley), suggests that the emission-line and far-infrared luminosity are also powered by young stars. Using the models of Hunter et al. and Hunter and Gallagher along with the mean far-infrared and extinction corrected $\mathrm{H} \alpha$ luminosity of the FIRGs yields an average total star formation rate of $\sim 70 \mathrm{M}_{\odot} \mathrm{yr}^{-1}$.

We propose that a typical ICSS FIRG be visualized as a "supergiant" H II region: a galaxy whose interstellar medium is being ionized (on scale of $1-10 \mathrm{kpc}$ ) by UV radiation and mass outflows as a direct result of a circumnuclear burst of star formation. While relatively few $(\leq 10 \%)$ of the ICSS FIRGs are observed to be presently interacting with a conspicuous companion galaxy, the great majority $(\sim 80 \%)$ are strongly distorted in continuum morphology. This suggests that our IR selection criteria are preferentially selecting galaxies that have recently undergone a merger.
\end{abstract}

Subject headings: galaxies: photometry — galaxies: structure — infrared: sources — nebulae: H II regions

\section{INTRODUCTION}

A major result of the $I R A S$ mission was the discovery of a class of powerful far-infrared galaxies (FIRGs) having farinfrared luminosities, $L_{\mathrm{FIR}}=10^{10}-10^{12} L_{\odot}$, and far-infrared to blue luminosity ratios $L_{\mathrm{FIR}} / L_{B}=10-100$. The far-infrared radiation from these galaxies is believed to be primarily thermal emission from heated dust grains. The optical spectra of the FIRGs are almost always dominated by emission lines. The most prominent of these lines are $\mathrm{H} \alpha,[\mathrm{N}$ II] $\lambda \lambda 6548,6583$, [S II] $\lambda \lambda 6717,6731,[\mathrm{O} \mathrm{I}] \lambda 6300, \mathrm{H} \beta$, and [O III] $\lambda \lambda 4959,5007$. The sizes, morphologies, and overall luminosities of the

\footnotetext{
${ }^{1}$ Visiting Observer, Kitt Peak National Observatory, National Optical Astronomy Observatory, operated by AURA, Inc. under contract with the National Science Foundation.
}

emission-line nebulae are a probe of the FIRG interstellar media. As such, they shed valuable light on the nature of the energy source lurking behind the enhanced activity that readily manifests itself in the far-infrared.

Prior to the present investigation, continuum-subtracted narrow-band images existed for only a handful of these powerful far-infrared galaxies. Nonetheless, it was clear that their emission-line morphologies were nothing like the $\mathrm{H}$ II regiondominated disks of normal spiral galaxies. Studies of Arp 220 and NGC 6240 have revealed large scale (tens of $\mathrm{kpc}$ ) intricate emission-line nebulosities (Heckman, Armus, and Miley 1987). Narrow-band imaging of the closest FIRG, M82, has uncovered a complex filamentary nebula (McCarthy, Heckman, and van Breugel 1987).

In an attempt to determine whether such spectacular mor- 
phology is common among the FIRGs as a class, we have undertaken a narrow-band $(\mathrm{H} \alpha+[\mathrm{N} \mathrm{II}])$ imaging survey of 30 galaxies having spectral indices, $\alpha \leq-1.5$ between 25 and 60 $\mu \mathrm{m}$, and $\alpha \geq-0.5$ between 60 and $100 \mu \mathrm{m}$ (where $\left.S_{v} \propto v^{\alpha}\right)$. The 30 galaxies that make up this infrared color-selected sample (ICSS) span both a large range in redshift $(z=0.001-0.169)$ and far-infrared luminosity $\left(L_{\text {FIR }} \sim 10^{10}-10^{12} L_{\odot}\right)$. For comparison purposes we also present narrow-band $(\mathrm{H} \alpha+[\mathrm{N} \mathrm{II}])$ images of 10 FIRGs selected to be bright at $60 \mu \mathrm{m}$ but not chosen with respect to far-infrared color.

\section{OBSERVATIONS AND DATA REDUCTION}

All galaxies presented in this paper (except for Arp 220 and NGC 6240) were imaged using the Cassegrain focus (f/7.5) CCD system with the T12 chip on the KPNO $2.1 \mathrm{~m}$ telescope. The images of Arp 220 and NGC 6240 were created using the prime focus (f/2.8) with the T12 chip on the KPNO $4 \mathrm{~m}$ telescope. Narrow-band filters centered on $\mathrm{H} \alpha+[\mathrm{N}$ II $]$, and a broad-band Mould $R$ filter were used for this imaging. Standard stars from Oke (1974) were used to flux-calibrate the galaxy images. Dome flat-fields were used to remove the pixelto-pixel variations of the chip, and typically more than one galaxy exposure was averaged together to avoid nonlinearity in the CCD. To reduce readout noise, adjacent columns and rows were binned together prior to read-out creating an effective pixel size of 0.38 for the KPNO $2.1 \mathrm{~m}$ telescope data, and 0 ".60 for the KPNO $4 \mathrm{~m}$ data. The respective fields-of-view were $2^{\prime} .5 \times 2^{\prime} .5$ and $4^{\prime} \times 4^{\prime}$.

To create a pure emission-line image for each galaxy, we performed the following reductions in the NRAO's AIPS data reduction package, except where explicitly noted. First, we subtracted the sky background from each pixel, determined by taking the average value in regions of the image devoid of stars or galaxies. For frames wherein the sky level varied systematically across the chip, a low-order polynomial was used to fit and subtract this variation on a row-by-row basis using the task Background in the NOAOs IRAF package. The continuum contribution to each narrow-band image was removed by subtracting a flux-scaled version of the corresponding broadband image. This resulted in an image of each galaxy in the light of $\mathrm{H} \alpha+[\mathrm{N} \mathrm{II}]$ alone. Successful continuum subtraction required the alignment of the two filter images and determination of the proper scale factor. Alignment of the broad and narrow-band frames was accomplished by fitting twodimensional Gaussians to at least three well-separated stellar images. The broad-band frame was then shifted by the proper amount in $x$ and $y$ (typically 1-2 pixels). The scale factor for subtraction was empirically determined so that stellar fluxes would become negligible in the final, pure emission-line image. Unfortunately, the point-spread functions in the broad-band frames were slightly larger than in the narrow-band frames. This may have been due to differences in the detector-to-filter distances (the pedestal focus) for the narrow- and broad-band filters. To compensate for this, we required the mean flux to become negligible within a box surrounding each stellar image, chosen large enough to encompass the stellar "halos" caused by this point-spread function difference.

Using the broad-band $R$ images to subtract out the continuum from the narrow-band images introduces added uncertainty because of the presence of emission lines within the $R$ bandpass. This will cause an oversubtraction of the narrowband images, and thus a slight underestimate of the $\mathrm{H} \alpha+[\mathrm{N} \mathrm{II}]$ fluxes. We have calculated the correction factor for each galaxy using the optical nuclear spectra from Armus, Heckman, and Miley (1989). The $\mathrm{H} \alpha+[\mathrm{N}$ II $]$ fluxes we have measured need to be multiplied by a typical value of 1.08 . These factors have not been applied to the data, instead serving as an indication of one type of uncertainty in the tabulated fluxes.

The image processing described above resulted in the generation of the 40 emission-line $(\mathrm{H} \alpha+[\mathrm{N} \mathrm{II}])$ images presented in Figure 1. The lowest level in the contour plots represents an isophotal level of $10^{-16}$ ergs $\mathrm{cm}^{-2} \mathrm{~s}^{-1} \operatorname{arcsec}^{-2}$, after having an atmospheric, a Galactic (Burstein and Heiles 1982; Savage and Mathis 1979), and a cosmological $\left[(1+z)^{4}\right]$ correction applied to the calibrated isophotal fluxes. These corrections give us a fixed isophotal level in the rest frame of the galaxy. Each level displayed in the contour plots represents an increase in flux by a factor of 2 above the previous level. For clarity, the lowest contours of the following galaxies are not at the $10^{-16}$ ergs $\mathrm{cm}^{-2} \mathrm{~s}^{-1}$ arcsec ${ }^{-2}$ level but are instead (in ergs $\mathrm{cm}^{-2} \mathrm{~s}^{-1}$ $\left.\operatorname{arcsec}^{-2}\right)$ : M82 $\left(10^{-15}\right)$, Arp $220\left(4 \times 10^{-17}\right)$, NGC 6240 $\left(6 \times 10^{-17}\right)$, and NGC 660 , NGC 2146, NGC 3079, NGC 4102 , and NGC $4536\left(2 \times 10^{-16}\right)$. Nuclear $\mathrm{H} \alpha+[\mathrm{N}$ II $]$ fluxes measured within a $2 \times 2 \mathrm{kpc}$ box and corrected for atmospheric extinction are given in column (6) of Tables 1 and 2. Total $\mathrm{H} \alpha+[\mathrm{N}$ II $]$ fluxes measured out to an isophotal level of $10^{-16}$ ergs $\mathrm{cm}^{-2} \mathrm{~s}^{-1} \operatorname{arcsec}^{-2}$ in the galaxy rest frame, and corrected for atmospheric extinction are listed in column (7) of Tables 1 and 2 . The nuclear and total $\mathrm{H} \alpha+[\mathrm{N}$ II $]$ luminosities (in ergs $\mathrm{s}^{-1}$ ) derived from these fluxes, corrected for Galactic extinction, are given in Tables 1 and 2 (cols. [8] and [9], respectively). Throughout this paper we use $H_{0}=75 \mathrm{~km} \mathrm{~s}^{-1}$ $\mathrm{Mpc}^{-1}$, and $q_{0}=0$. Since we have included $\mathrm{H} \alpha+[\mathrm{N}$ II $]$ flux only down to the $10^{-16}$ ergs $\mathrm{cm}^{-2} \mathrm{~s}^{-1} \operatorname{arcsec}^{-2}$ level, the values we give for $L_{\text {TOт }}$ will be a slight underestimate (typically a few percent) of the "true" total $\mathrm{H} \alpha+[\mathrm{N} \mathrm{II}]$ emission. Our total and nuclear emission-line fluxes are accurate therefore to $\sim 10 \%$, with the tabulated values preferentially being underestimates.

The accompanying $R$ band images are presented to show the general continuum (stellar) morphology of these galaxies. The lowest contour in these plots is at 4 times the rms in the sky, with each successive level representing an increase in flux by a factor of 2 over the previous level. The total, apparent $R$ band magnitudes were derived by first clipping the broad-band images at an isophotal level of $24 \mathrm{mag} \operatorname{arcsec}^{-2}$ (in the rest frame of the galaxy), and then measuring the flux (corrected for atmospheric and Galactic extinction) within a box encompassing the galaxy. The "nuclear" apparent $R$ band magnitudes were calculated by measuring the flux within the same aperture used to measure the nuclear $\mathrm{H} \alpha+[\mathrm{N}$ II] fluxes. The total and nuclear absolute $R$ band magnitudes (cols [11] and [12] of Tables 1 and 2, respectively) were then derived using the following formula appropriate for $q_{0}=0$ :

$$
M=m-42.384+5 \log (h)-5 \log [z(1+z / 2)]-K,
$$

where $h=H_{0} / 100 \mathrm{~km} \mathrm{~s}^{-1} \mathrm{Mpc}^{-1}, m$ is the apparent magnitude, and $K$ is the $K$-correction. In all cases an $h=0.75$, and a $K$-correction appropriate to an Sbc galaxy were used (Coleman, Wu, and Weedman 1980). We have not corrected the $R$ band magnitudes for the inclusion of emission lines in the broad-band filter. From the measurements of the $\mathrm{H} \alpha+[\mathrm{N} \mathrm{II}]$ equivalent widths (see below), we conclude that this correction is negligible outside of our $2 \mathrm{kpc}$ nuclear aperture, and in most 


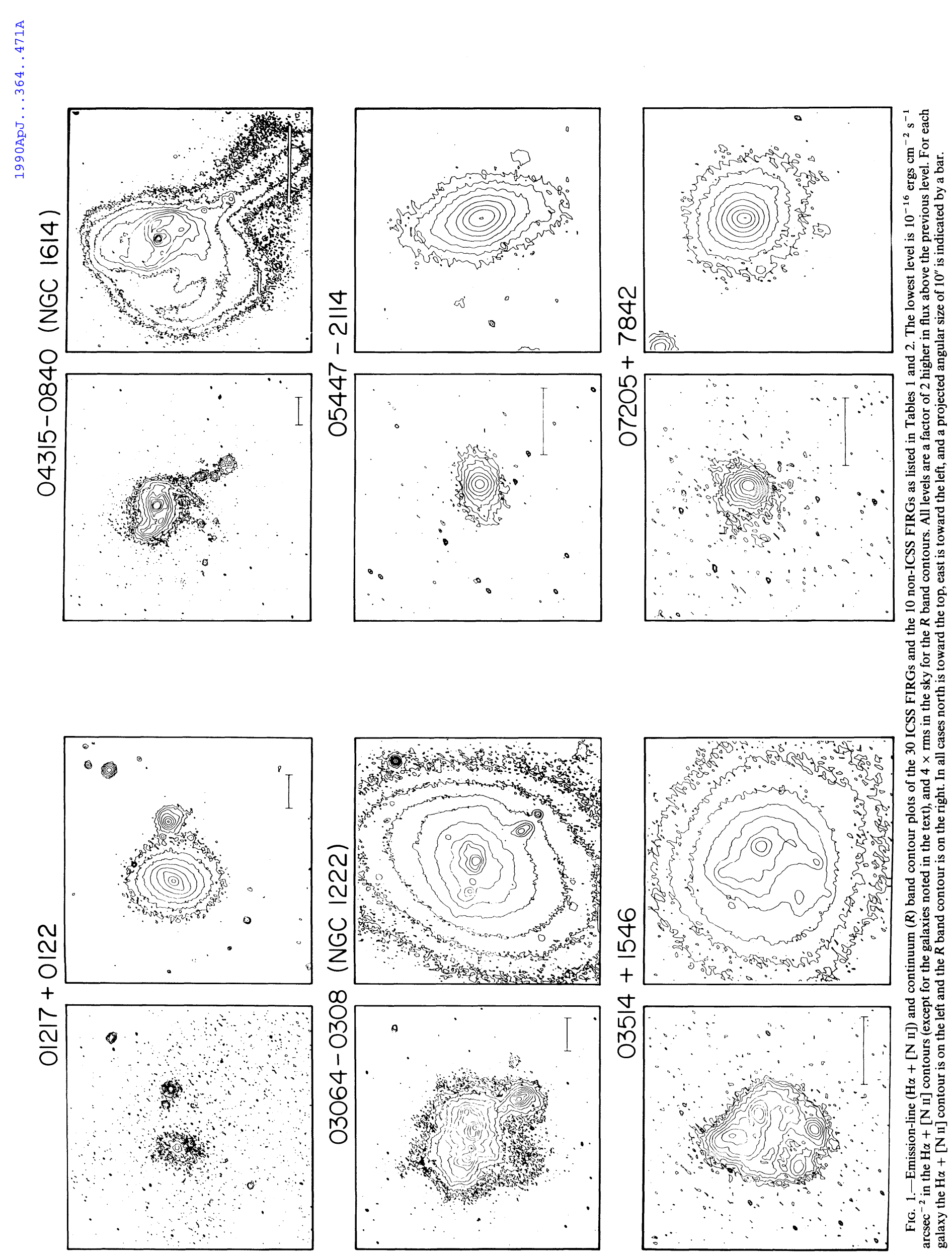



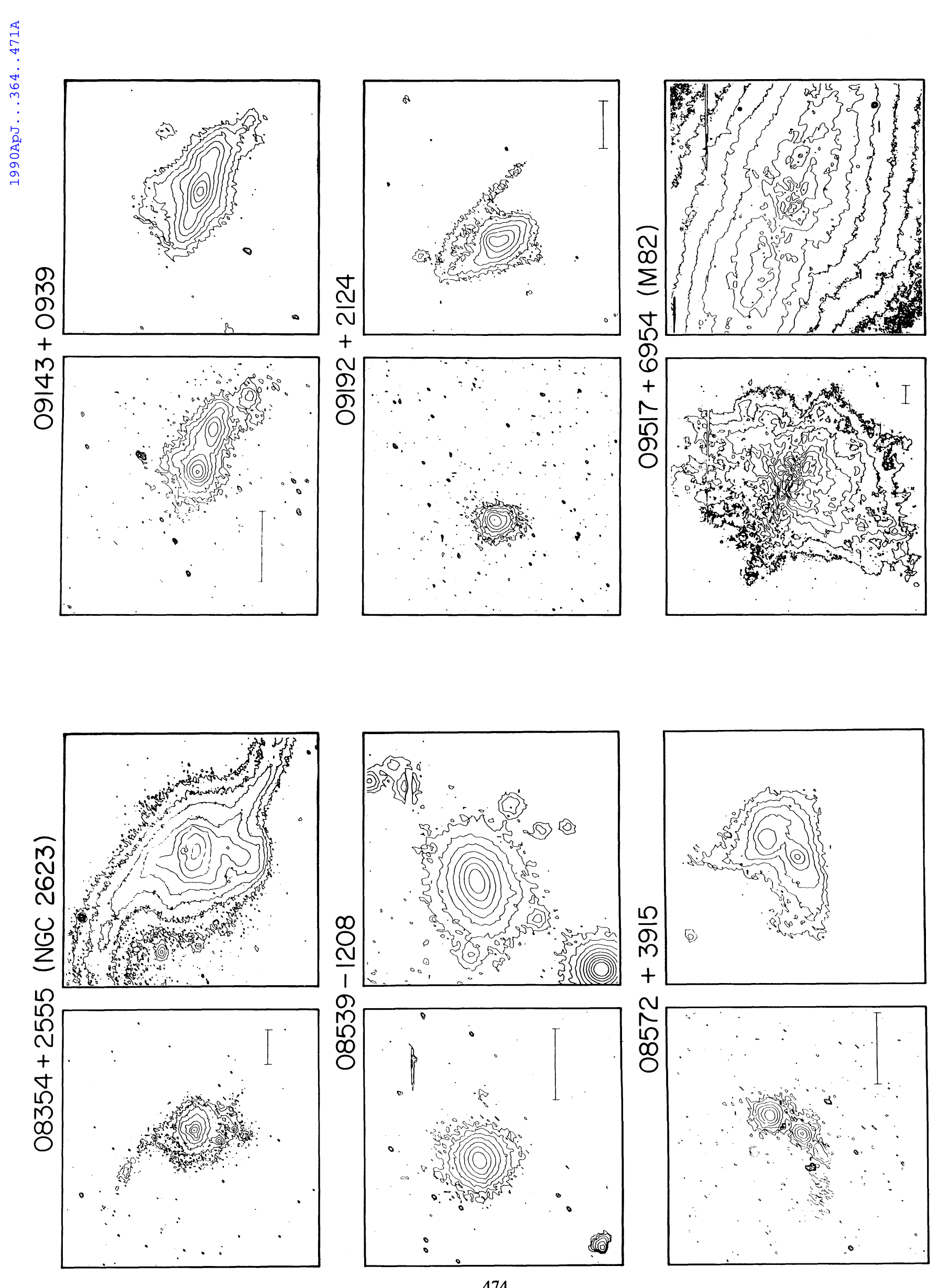

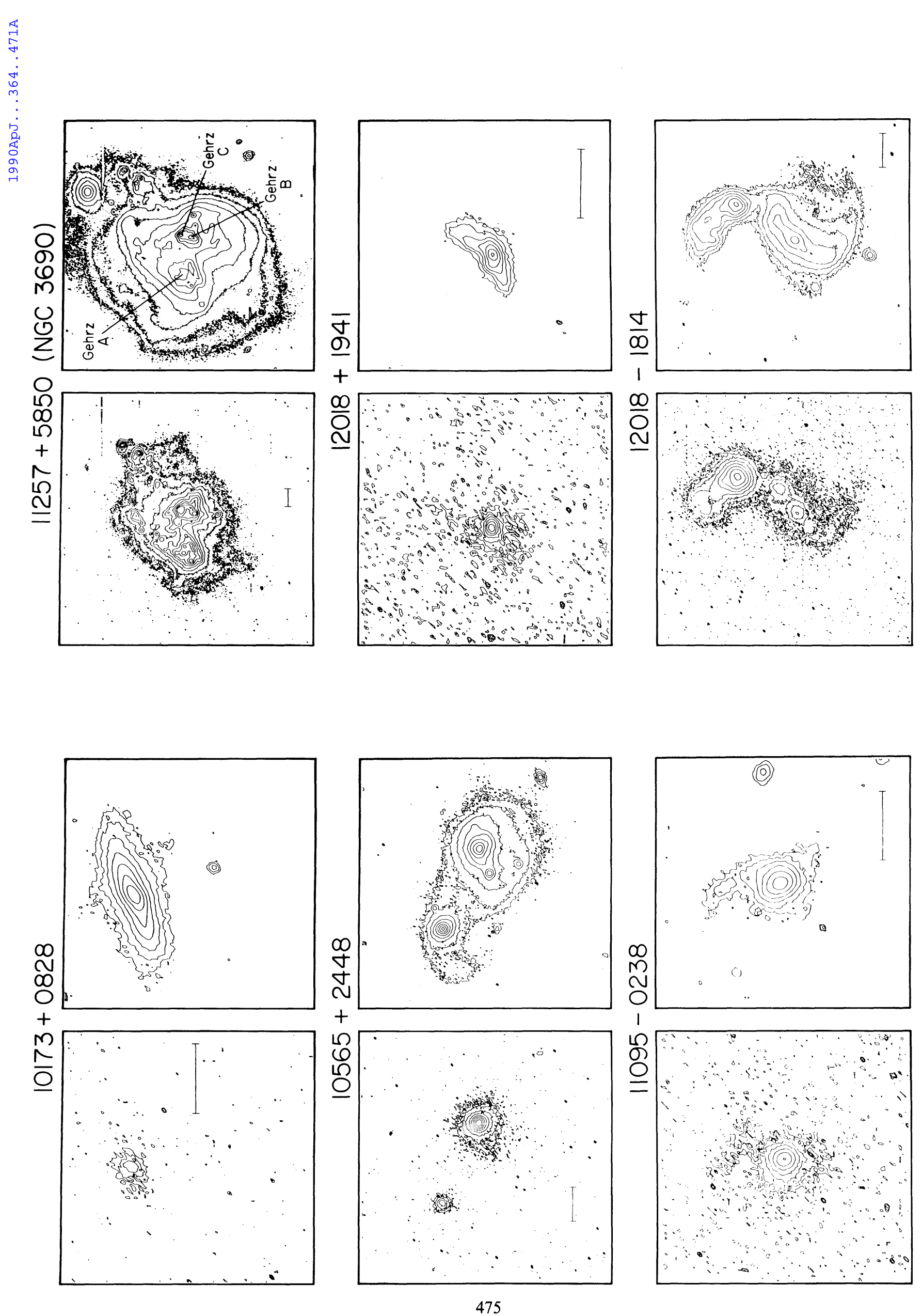

(C) American Astronomical Society - Provided by the NASA Astrophysics Data System 

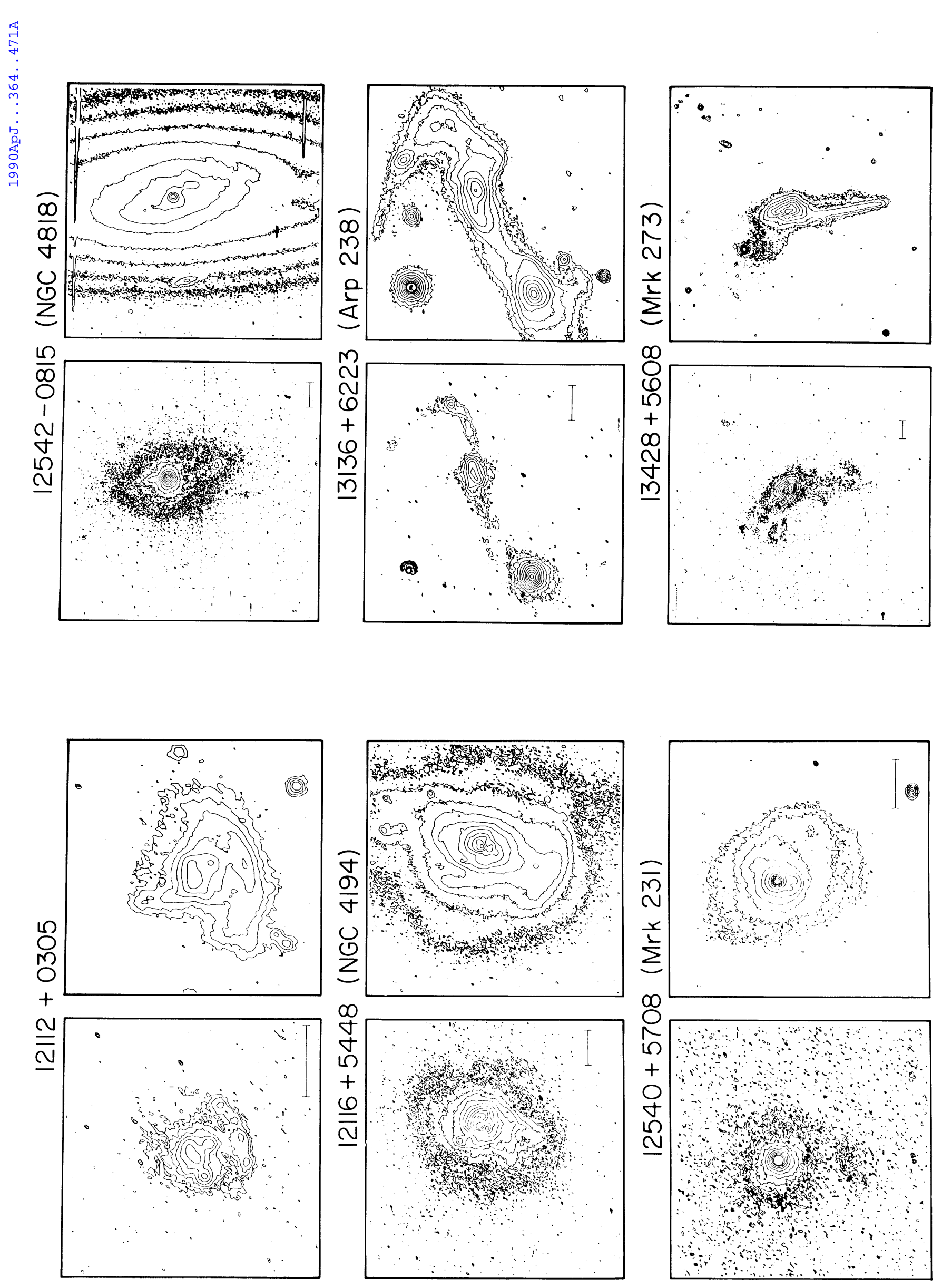

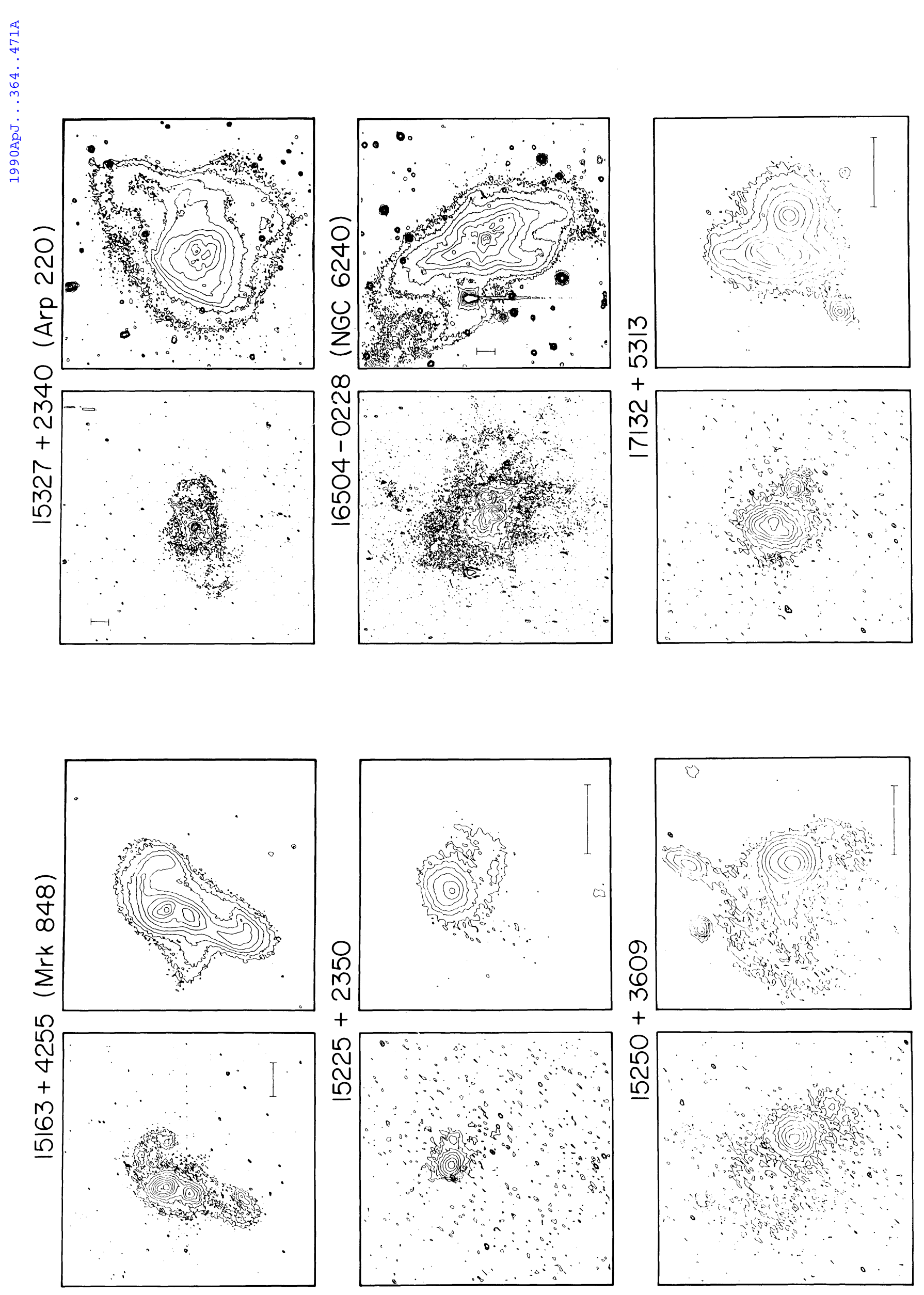

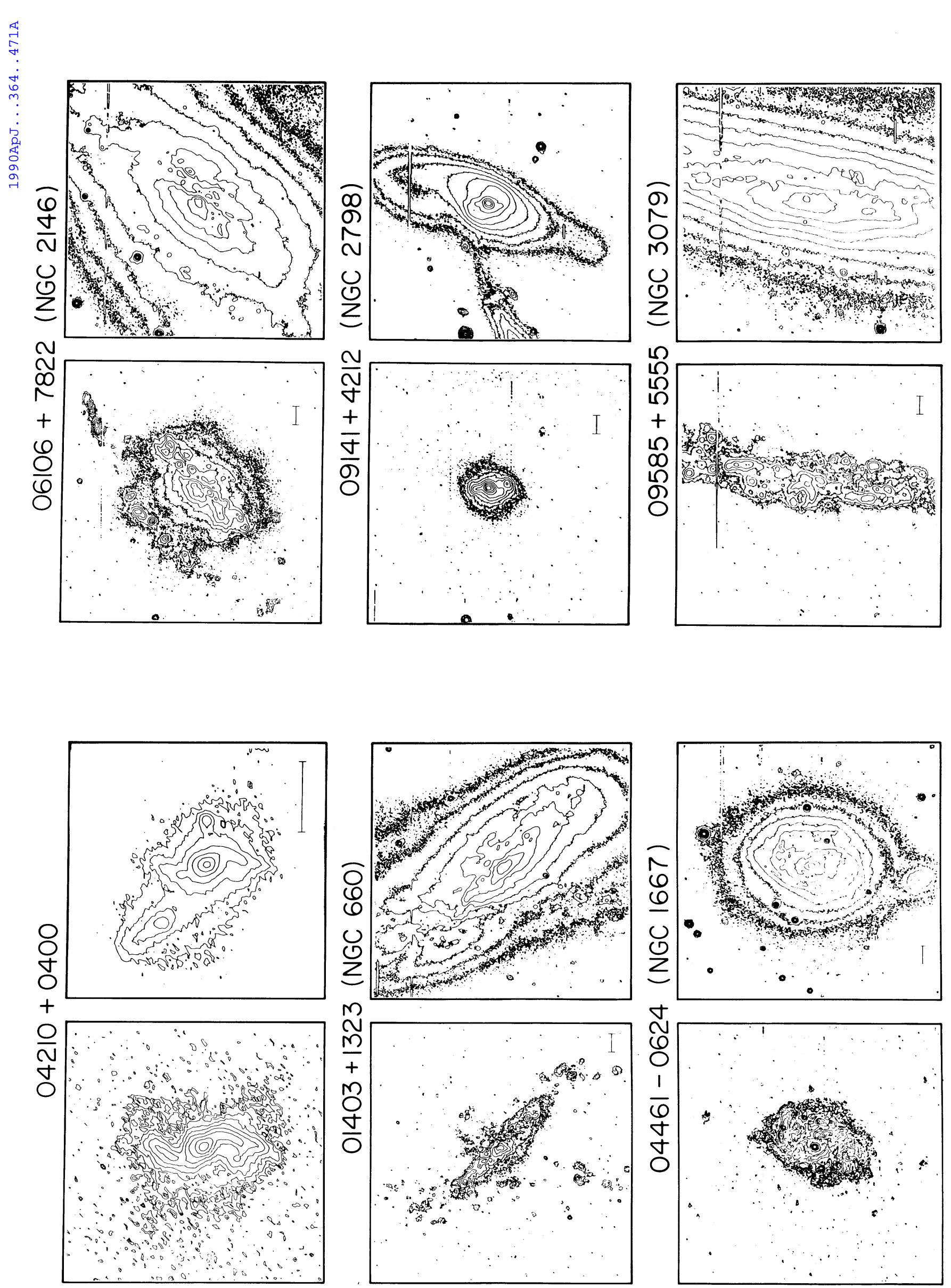

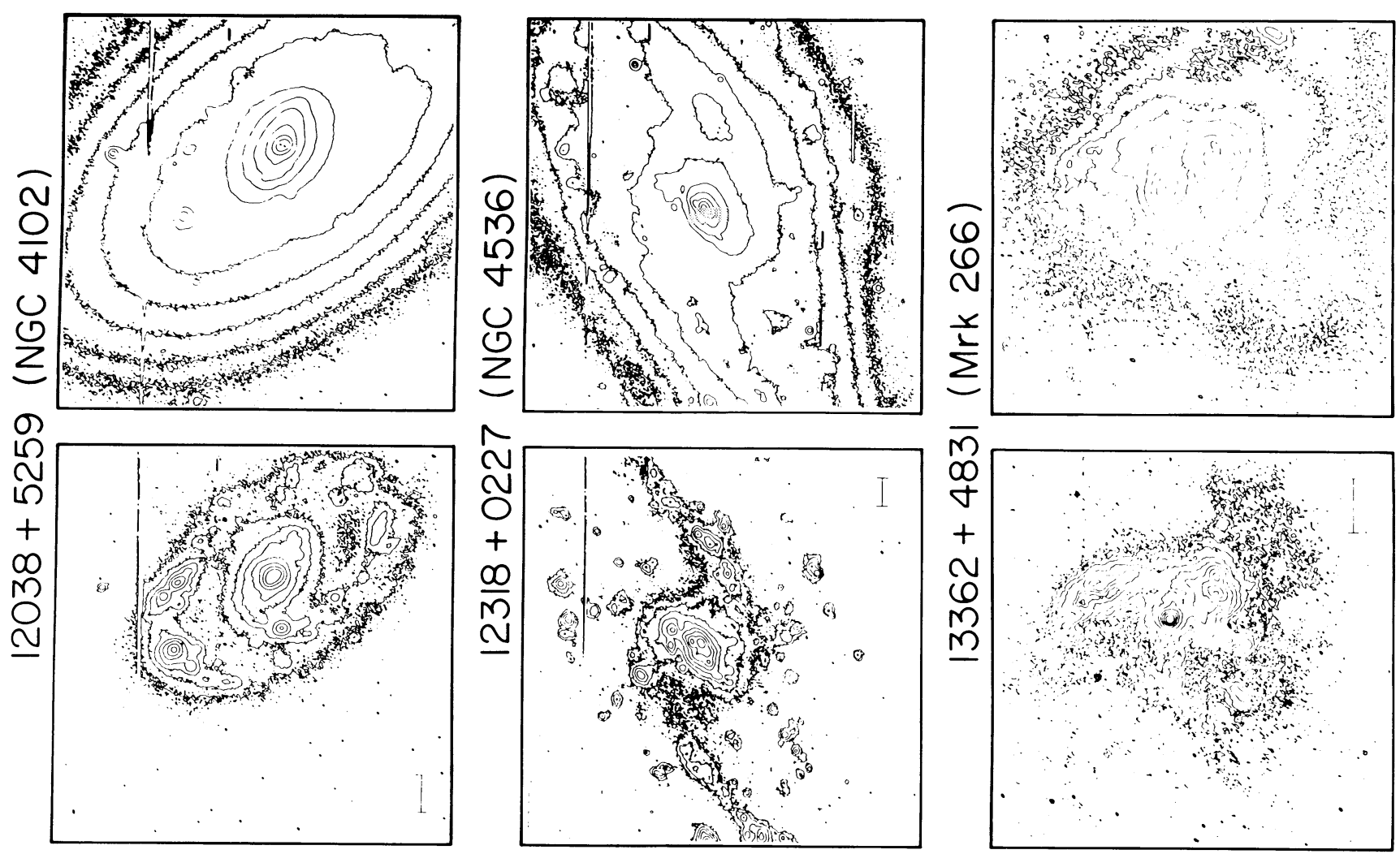

479

(C) American Astronomical Society - Provided by the NASA Astrophysics Data System 


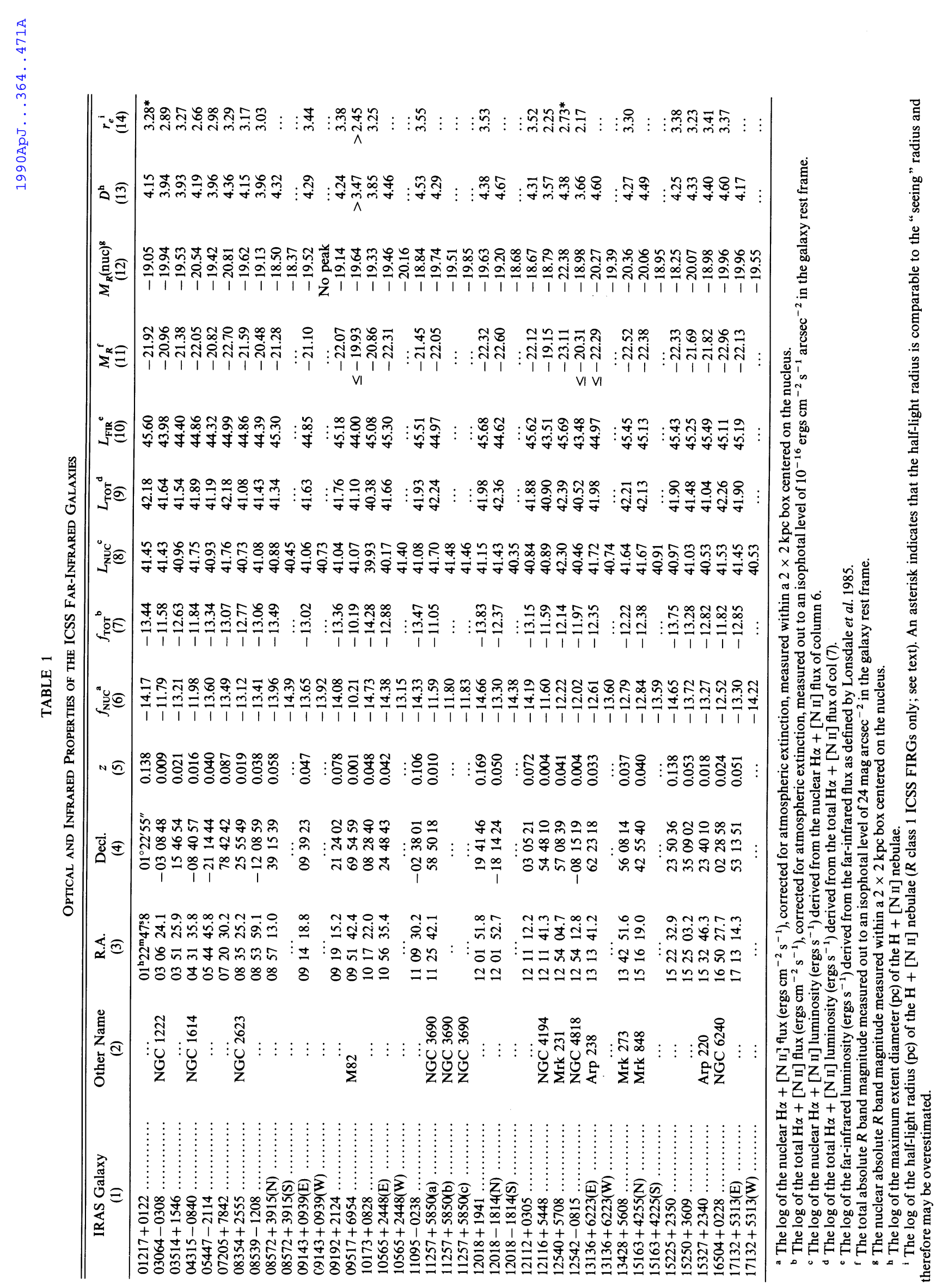


TABLE $2^{\mathrm{a}}$

Optical and Infrared Properties of the Non-ICSS Far-INFrared Galaxies

\begin{tabular}{|c|c|c|c|c|c|c|c|c|c|c|c|c|}
\hline $\begin{array}{l}\text { IRAS Galaxy } \\
\text { (1) }\end{array}$ & $\begin{array}{l}\text { Other Name } \\
\text { (2) }\end{array}$ & $\begin{array}{l}\text { R.A. } \\
\text { (3) }\end{array}$ & $\begin{array}{l}\text { Decl. } \\
(4)\end{array}$ & $\begin{array}{c}z \\
(5)\end{array}$ & $\begin{array}{c}f_{\text {NUC }} \\
(6)\end{array}$ & $\begin{array}{c}f_{\text {TOT }} \\
(7)\end{array}$ & $\begin{array}{c}L_{\mathrm{NUC}} \\
(8)\end{array}$ & $\begin{array}{l}L_{\text {TOT }} \\
(9)\end{array}$ & $\begin{array}{l}L_{\mathrm{FIR}} \\
(10)\end{array}$ & $\begin{array}{l}M_{R} \\
(11)\end{array}$ & $\begin{array}{l}M_{R}(\text { nuc }) \\
\quad(12)\end{array}$ & $\begin{array}{c}D \\
(13)\end{array}$ \\
\hline $04210+0400(\mathrm{E})$ & $\cdots$ & $04^{\mathrm{h}} 21^{\mathrm{m}} 01^{\mathrm{s}} \cdot 6$ & $04^{\circ} 00^{\prime} 58^{\prime \prime}$ & 0.046 & -15.36 & -12.61 & 39.38 & 42.13 & 44.19 & -21.55 & -17.64 & 4.30 \\
\hline $04210+0400(\mathrm{~W})$ & $\ldots$ & & & & -13.21 & $\ldots$ & 41.53 & & & $\ldots$ & -19.39 & $\cdots$ \\
\hline $01403+1323 \ldots \ldots \ldots \ldots$ & NGC 660 & $\begin{array}{lll}01 & 40 & 21.0\end{array}$ & 132318 & 0.003 & -12.19 & -11.89 & 40.07 & 40.37 & 43.68 & & -18.66 & 3.87 \\
\hline $04461-0624(N) \ldots \ldots \ldots$ & NGC 1667 & $0446 \quad 10.2$ & -062424 & 0.015 & -12.99 & -12.05 & 40.70 & 41.65 & 44.20 & & -20.28 & 4.30 \\
\hline $04461-0624(S) \ldots \ldots \ldots$ & & $\ldots$ & $\ldots$ & $\ldots$ & -14.66 & $\ldots$ & 39.03 & $\ldots$ & $\ldots$ & $\ldots$ & -17.73 & $\ldots$ \\
\hline $06106+7822 \ldots \ldots \ldots \ldots$ & NGC 2146 & 061045.0 & 782230 & 0.003 & -11.55 & -11.31 & 40.79 & 41.03 & 44.18 & $\ldots$ & -19.23 & 3.98 \\
\hline $09141+4212(E) \ldots \ldots \ldots$. & NGC 2798 & 091409.6 & 421242 & 0.006 & $\geq-14.00$ & $\geq-11.78$ & $\geq 38.84$ & $\geq 41.06$ & 43.83 & & & \\
\hline $09141+4212(W) \ldots \ldots \ldots$ & & 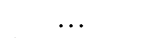 & 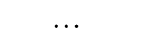 & $\ldots$ & -11.85 & $\ldots$ & 40.99 & .. & $\ldots$ & $\ldots$ & -19.46 & $\ldots$ \\
\hline $09585+5555 \ldots \ldots \ldots \ldots$ & NGC 3079 & $\begin{array}{lll}09 & 58 & 34.8\end{array}$ & 555524 & 0.004 & -12.23 & $\geq-11.59$ & 40.26 & $\geq 40.90$ & 43.88 & & -18.97 & $>4.07$ \\
\hline $12038+5259 \ldots$ & NGC 4102 & 120351.6 & 525918 & 0.003 & -11.66 & -11.43 & 40.58 & 40.81 & 43.69 & & -19.32 & 3.74 \\
\hline $12318+0277 \ldots \ldots \ldots \ldots \ldots$ & NGC 4536 & 123154.0 & 022742 & 0.006 & -11.79 & $\geq-11.41$ & 40.05 & $\geq 41.43$ & 44.02 & $\ldots$ & -19.64 & $>4.29$ \\
\hline $13362+4831(\mathrm{~N}) \ldots \ldots \ldots$ & Mrk 266 & 133615.0 & 483154 & 0.028 & -12.55 & -11.90 & 41.64 & 42.29 & 44.76 & -22.85 & -20.48 & 4.50 \\
\hline $13362+4831(\mathrm{~S}) \ldots \ldots \ldots \ldots$ & & $\cdots$ & $\cdots$ & & -12.74 & $\ldots$ & 41.45 & $\ldots$ & $\ldots$ & $\ldots$ & -20.06 & $\cdots$ \\
\hline $15132+4223 \ldots \ldots \ldots \ldots \ldots$ & NGC 5900 & 151316.2 & 422342 & 0.009 & -13.25 & -12.60 & 39.95 & 40.58 & 43.84 & -20.15 & -18.60 & 4.06 \\
\hline
\end{tabular}

a See notes for Table 1 for explanation of columns.

cases $<8 \%$ in the nucleus. We estimate that our absolute $R$ band magnitudes are accurate (internally) to $\sim 0.10 \mathrm{mag}$.

The far-infrared luminosity $\left(L_{\text {FIR }}\right)$ calculated from the farinfrared flux as defined by Lonsdale et al. (1985) is listed in column 10.

\section{RESULTS}

\section{a) Morphology}

As evidenced by Figure 1, there is a wide range of morphological types among the $\mathrm{H} \alpha+[\mathrm{N} \mathrm{II}]$ emission-line nebulae of our sample galaxies. For example, we see the following:

1. Double-galaxy systems with $\mathrm{H} \alpha$ emission that surrounds each nucleus, and in some cases forms connecting bridges between the galaxies. A good example of this type of ICSS FIRG is $13136+6223$.

2. Single or multiple nucleus systems with large-scale filamentary structure (e.g., NGC 3690, NGC 6240). Sometimes these nebulae can appear totally chaotic on small scales as in the case of M82. The "frothy" nature of the M82 emission-line nebula may be particularly conspicuous, since it is the closest member of the sample.

3. Single galaxies with large scale (up to tens of $\mathrm{kpc}$ ) seemingly coherent features at low $\mathrm{H} \alpha$ levels (e.g., Arp 220 and perhaps Mrk 273).

4. Galaxies with a single dominant nucleus in $\mathrm{H} \alpha+[\mathrm{N}$ II $]$ plus giant $\mathrm{H}$ II regions or hot spots, but with little or no filamentary structure (e.g., NGC 1614, 03514+1546, NGC 2623).

5. Galaxies with emission-line nebulae whose brightness drops off smoothly from the nucleus with no evidence for giant $\mathrm{H}$ II regions, or filamentary structure (e.g., 05447-2114, 08539-1208).

It is interesting to note that there is not one ICSS FIRG with an $\mathrm{H} \alpha+[\mathrm{N} \mathrm{II}]$ emission-line morphology resembling a normal spiral galaxy (we will discuss this further in $\S I V$ ).

The $\mathrm{H} \alpha$ emission often seems to follow the $R$ band emission, with the position angle of maximum extent aligned. Some ICSS galaxies, however, appear to be elongated in $\mathrm{H} \alpha+[\mathrm{N} \mathrm{II}]$ perpendicularly to the continuum major axis. Of the seven flattest ICSS FIRGs (those having a projected major-to-minor axis ratio $\geq 2.0$ in the $R$ band image), six show evidence for $\mathrm{H} \alpha+[\mathrm{N} \mathrm{II}]$ emission along the minor axis. The $\mathrm{H} \alpha+[\mathrm{N} \mathrm{II}]$ contours in these six galaxies are either (at high and/or low surface brightness levels) more circular than (e.g., 12542-0815,
$10173+0828$ ), or clearly perpendicular to (e.g., $05447-2114$, M82, NGC 6240), the $R$ band continuum contours. Since these six galaxies are apparently highly inclined (flat), the $\mathrm{H} \alpha+[\mathrm{N} \mathrm{II}]$ isophotes may represent ionized gas that is extended along the minor axis (i.e., along the rotation axis or out of the disk of the galaxy). The seventh galaxy with a projected major-to-minor axis ratio $\geq 2.0,09143+0939$, has a morphology that is similar in $R$ and $\mathrm{H} \alpha+[\mathrm{N}$ II $]$. For the less inclined (more nearly face-on) members of our sample with $\mathrm{H} \alpha+[\mathrm{N} \mathrm{II}]$ isophotes at right angles to their $R$ band continuum isophotes (e.g., $07205+7842$ and $08539-1208$ ), the true spatial relationship between the stars and the ionized gas is less clear. In addition, the non-ICSS FIRGs NGC 660, NGC 2146, NGC 3079, and NGC 5900 (four nearly edge-on spirals) have faint $\mathrm{H} \alpha+\left[\mathrm{N}_{\text {II }}\right]$ features that lie along their minor axis (see below).

The 10 other galaxies not selected for their IR color are predominantly spirals showing the $\mathrm{H} \alpha$ signature of $\mathrm{H}$ II regions following along the spiral arms (e.g., NGC 1667, NGC 4536). The galaxy Mrk 266, however, exhibits a multiple nucleus and extended filamentary structure rivaling what is seen in any of the ICSS FIRGs.

\section{b) Size}

The ICSS FIRG emission-line nebulae are large. Most of the galaxies do not have symmetrical nebular shapes (see below) that lend themselves easily to the definition of a major and minor axis. Instead, we have measured the maximum extent (diameter) of the nebulae out to an isophotal level of $10^{-16}$ ergs $\mathrm{cm}^{-2} \mathrm{~s}^{-1} \operatorname{arcsec}^{-2}$ (in the rest frame of the galaxy). The ICSS FIRGs have nebulae that range in angular size from about 7" up to greater than $150^{\prime \prime}$ in diameter. The corresponding linear dimensions are impressive, ranging from about $3 \mathrm{kpc}$ up to nearly $50 \mathrm{kpc}$. The distribution of nebular diameters, $D$, is shown in Figure $2 a$ and listed in column (13) of Table 1 and Table 2 (for the non-ICSS FIRGs). The mean nebular diameter is $\sim 16 \mathrm{kpc}$ for the IR color-selected FIRGs. Emissionline gas in the ICSS FIRGs is clearly not confined solely to the circumnuclear regions.

The half-light radius, $r_{e}$, ranges from $\sim 150$ to $3500 \mathrm{pc}$ for the ICSS FIRG emission-line nebulae, with a mean $\log r_{e}=$ $3.11(\sim 1.3 \mathrm{kpc})$. The distribution of $\log r_{e}$ is shown in Figure $2 b$. Note that these values of $r_{e}$ apply only to the ICSS FIRGs 

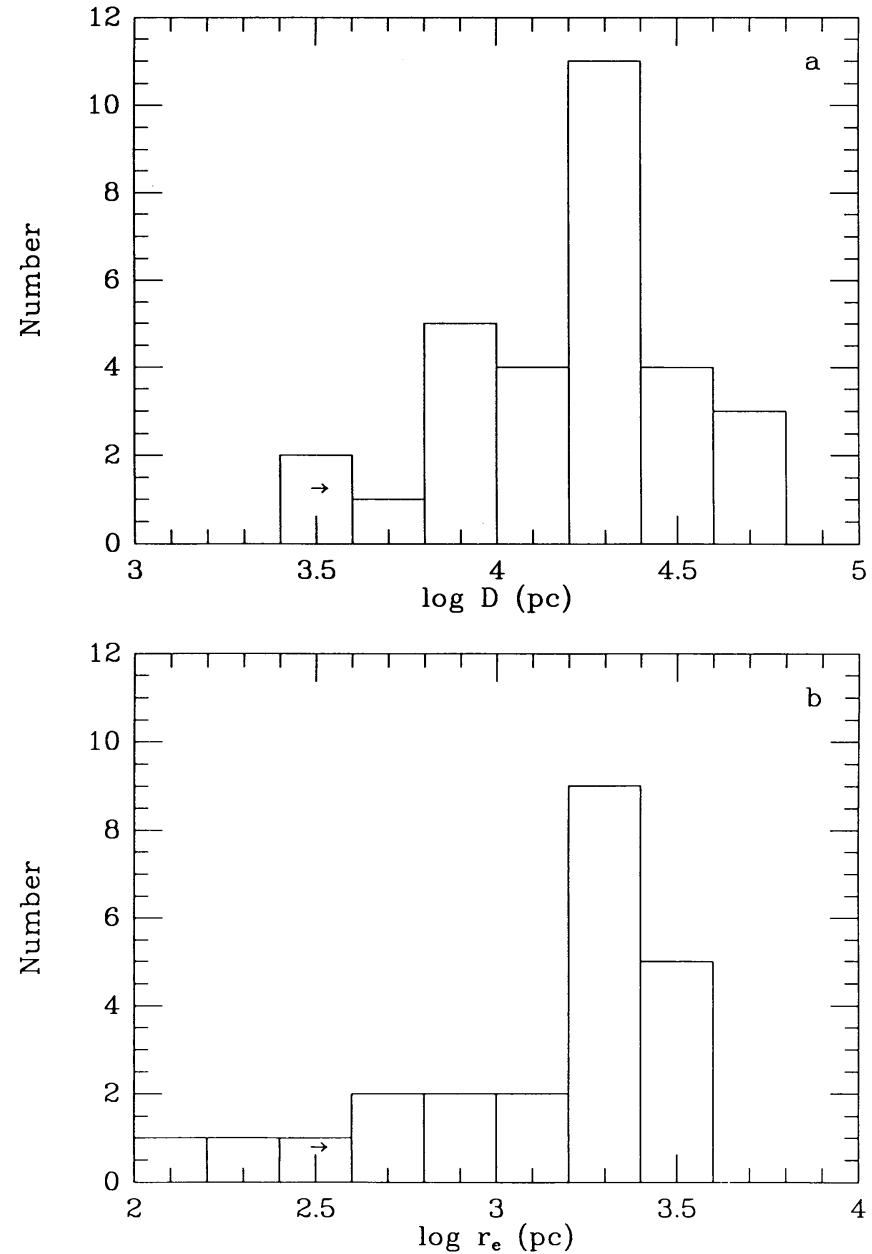

Fig. 2.-Distribution of (a) the log maximum extent, $D(\mathrm{pc})$ in $\mathrm{H} \alpha+[\mathrm{N} \mathrm{II}]$ at the $10^{-16} \mathrm{ergs} \mathrm{cm}^{-2} \mathrm{~s}^{-1} \operatorname{arcsec}^{-2}$ level for all the ICSS FIRGs, and $(b)$ the $\log$ of the $\mathrm{H} \alpha+[\mathrm{N} \mathrm{II}]$ half-light radius, $r_{e}$ (pc) of the $R$ class 1 (see text) ICSS FIRGs. Arrows denote lower limits to $D, r_{e}$.

that are single-galaxy systems $(\sim 75 \%$ of the sample, see below), and they are derived from the $\mathrm{H} \alpha+[\mathrm{N}$ II $]$ intensity, azimuthally averaged over concentric circles centered on the nucleus of the galaxy.

\section{c) Luminosity}

We have measured total $\mathrm{H} \alpha+[\mathrm{N}$ II $]$ luminosities (out to a corrected surface brightness level of $10^{-16} \mathrm{ergs}^{\mathrm{cm}} \mathrm{cm}^{-2} \mathrm{~s}^{-1}$ $\operatorname{arcsec}^{-2}$ ) for all the galaxies displayed in Figure 1. Note that we have not corrected these luminosities for extinction internal to the galaxies (a correction that could be substantial given a mean nuclear extinction at $\mathbf{H} \beta$ of $\sim 2.5 \mathrm{mag}$; see Armus, Heckman, and Miley 1989). For our ICSS FIRGs $40.38 \leq \log$ $L_{\text {ToT }}\left(\right.$ ergs $\left.\mathrm{s}^{-1}\right) \leq 42.40$, with a mean $\log L_{\text {TOT }}=41.67$ $(\sigma=0.53)$, and $43.48 \leq \log L_{\mathrm{FIR}} \leq 45.68$, with a mean $\log$ $L_{\text {FIR }}=44.94(\sigma=0.61)$. The distribution of $\log L_{\text {TOT }}$ is shown in Figure 3. The galaxies in Table 2 (non-ICSS FIRGs) have a mean $\log L_{\text {TOT }}=41.23(\sigma=0.64)$, and a mean $\log L_{\mathrm{FIR}}=44.03$ $(\sigma=0.32)$. The ICSS FIRGs, then, have mean total $\mathrm{H} \alpha+[\mathrm{N}$ II $]$ and far-infrared luminosities that are larger than the ICSS FIRGs not selected with regard to far-IR color by factors of $\sim 2.8(98 \%$ confidence level) and $\sim 8.1(>99.5 \%$ confidence level), respectively.

Since many of our IR color-selected galaxies appear to have highly distorted morphologies that may be indicative of current or past interactions/mergers, we can compare these luminosities to an optically selected sample of interacting galaxies taken from Bushouse, Lamb, and Werner (1988, hereafter BLW). These systems were chosen from the Arp atlas (Arp 1966) and the UGC (Nilson 1973) to show strong tidal interactions while still displaying evidence for at least one stellar disk per pair. Four galaxies, NGC 1614, NGC 2623, NGC 3690 , and $13136+6223$ (Arp 238), are common to both the BLW (1988) sample, and our sample of ICSS FIRGs. To arrive at a "pure" $\mathrm{H} \alpha$ flux, BLW (1988) have corrected the observed $\mathrm{H} \alpha+[\mathrm{N}$ II $]$ flux by a factor of 0.75 , to account in a general way for the presence of the [N II] $\lambda \lambda 6548,6583$ lines. To place the optically selected interacting galaxies on our $\mathrm{H} \alpha+[\mathrm{N}$ II $]$ luminosity scale then, we have multiplied the $\mathrm{H} \alpha$ fluxes from BLW (1988) by a factor of 1.33. The loci of these galaxies, as well as a sample of isolated spiral and irregular galaxies from Kennicutt and Kent (1983, hereafter KK), are labeled alongside our ICSS FIRGs in Figure 4. The high end of the distribution of isolated galaxies is made up of predominantly giant $\mathrm{Sc}$ galaxies with an $L_{\text {Tот }}\left(\right.$ ergs $\left.\mathrm{s}^{-1}\right) \sim 41.7\left(H_{0}=75 \mathrm{~km} \mathrm{~s}^{-1}\right.$ $\left.\mathrm{Mpc}^{-1}\right)$. The infrared luminosity of our sample galaxies is greater than either the interacting galaxies, or the isolated galaxies (as expected due to our IR selection criteria). The mean $\log \mathrm{H} \alpha+\left[\mathrm{N}_{\mathrm{II}}\right]$ luminosities of the isolated and interacting galaxies are $L_{\text {Tот }}\left(\operatorname{ergs~} \mathrm{s}^{-1}\right)=40.92$, and $41.58(\sigma=0.43)$ respectively. While our IR color-selected FIRGs are clearly more luminous in $\mathrm{H} \alpha+[\mathrm{N}$ II] than the isolated galaxies, their total $\mathrm{H} \alpha+\left[\mathrm{N}_{\text {II }}\right]$ luminosities are similar to those of the optically selected interacting galaxies. Most $(\geq 75 \%)$ of our ICSS FIRGs, however, are not double-galaxy systems (see below), so a direct comparison to the total system $\mathrm{H} \alpha+[\mathrm{N}$ II $]$ luminosities of the optically selected interacting galaxies may not be appropriate (depending upon the distribution of the emissionline flux between the two galaxies in the BLW 1988 sample). Also, while there are eight ICSS FIRGs with $\log L_{\text {Tот }}>42.0$, the mean $\mathrm{H} \alpha+[\mathrm{N}$ II] luminosity is comparable to that found for the brightest Sc galaxies.

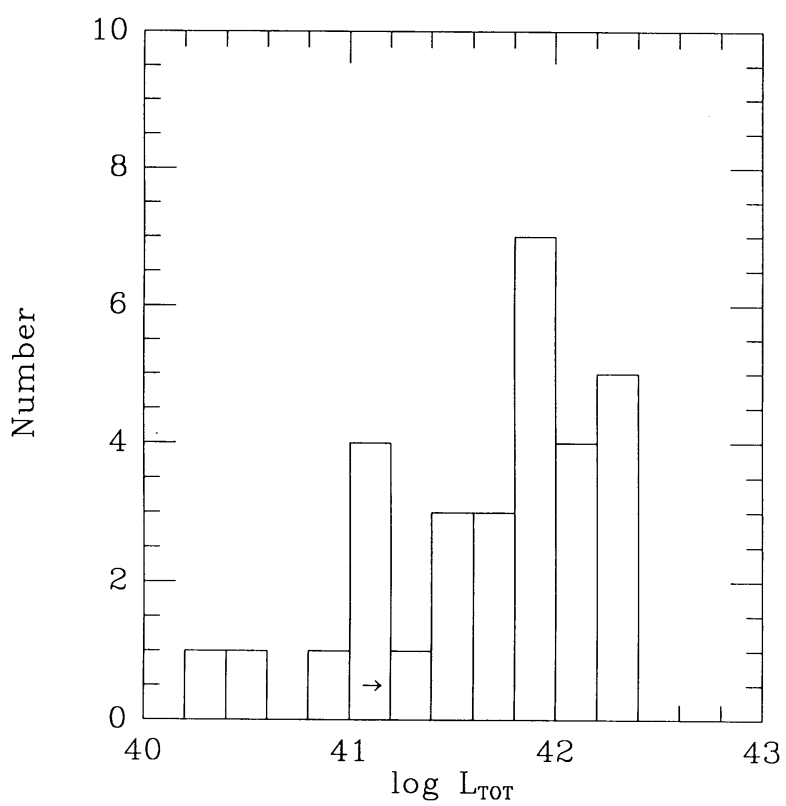

FIG. 3.-Distribution of the $\log$ of the total (defined in text) $\mathrm{H} \alpha+[\mathrm{N} \mathrm{II}]$ luminosity $\left(L_{\text {Tот }}\right)$, uncorrected for internal extinction, for the ICSS FIRGs. Arrow denotes a lower limit to $L_{\text {TOT }}$. 


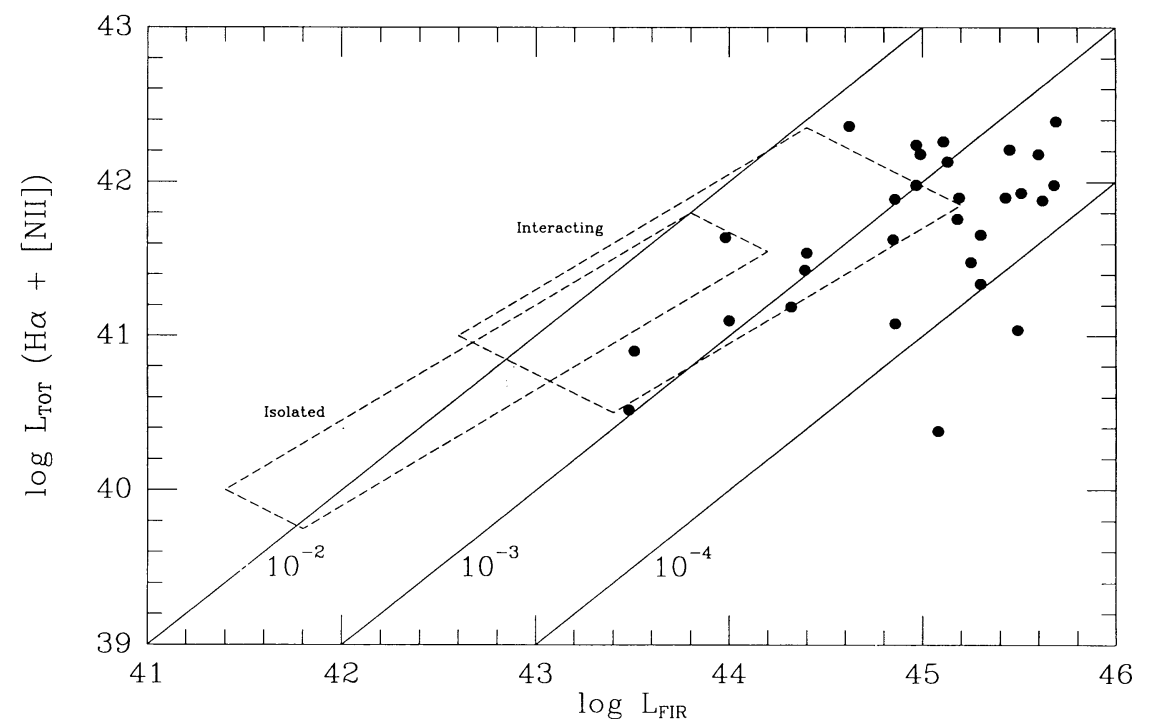

FIG. 4.-Plot of the far-infrared luminosity $\left(L_{\mathrm{FIR}}\right)$ against the total $\mathrm{H} \alpha+[\mathrm{N}$ II $]$ luminosity $\left(L_{\mathrm{TOT}}\right)$ of the ICSS FIRGs. Also plotted are the loci of interacting spiral galaxies taken from Bushouse, Lamb, and Werner (1988), and the loci of isolated spiral galaxies taken from Kennicutt and Kent (1983). The solid diagonal lines represent select ratios of $\mathrm{H} \alpha+[\mathrm{N}$ II $]$ to far-infrared luminosity. The $\mathrm{H} \alpha+[\mathrm{N}$ II $]$ luminosity is uncorrected for internal extinction.

Also plotted in Figure 4 are lines that correspond to various ratios of $L_{\text {TOT }} / L_{\text {FIR }}$. For the ICSS FIRGs $-4.5 \leq \log$ $\left(L_{\text {TOT }} / L_{\text {FIR }}\right) \leq-2.5$ with a mean $\log \left(L_{\text {TOT }} / L_{\text {FIR }}\right)=-3.27$ $(\sigma=0.56)$. The isolated galaxies typically have log $\left(L_{\text {Tот }} / L_{\text {FIR }}\right)=-2.0$ and the optically selected interacting galaxies typically have $-3.0 \leq \log \left(L_{\text {TOT }} / L_{\text {FIR }}\right) \leq-2.0$. The nonICSS FIRGs of Table 2 have a mean $\log \left(L_{\text {TOT }} / L_{\text {FIR }}\right)=-2.80$ $(\sigma=0.40)$. The scatter in $\log \left(L_{\text {TOT }} / L_{\text {FIR }}\right)$ for our ICSS FIRGs is even greater than that seen in the optically selected interacting galaxies. BLW (1988) attribute the scatter seen in the interacting galaxies to nonequilibrium star formation in the recent past. This is based upon the assumption that the far-infrared and $\mathrm{H} \alpha$ luminosities measure the average SFR over slightly different periods of time [ $L_{\mathbf{H} \alpha}$ arises primarily from ionization by young stars with main-sequence lifetimes $\leq 10^{7} \mathrm{yr}$, whereas $L_{\text {FIR }}$ has a contribution from warm $(50 \mathrm{~K})$ dust heated by current star formation (OB associations), as well as cooler $(25-30 \mathrm{~K})$ dust heated by the general interstellar radiation field (see Persson and Helou 1987 and Rowan-Robinson and Crawford 1989]. A large range in current-to-past SFR may therefore be responsible for the scatter about the linear fit that we see for the ICSS FIRGs in Figure 4.

An alternative explanation, however, is that the scatter we observe is due to the variable, and sometimes considerable, internal extinction evident in the optical spectra of the ICSS FIRGs. This would tend to move the points more or less vertically about the best-fit line in Figure 4 . It may be that a combination of nonequilibrium star formation and visual extinction is responsible for the increased scatter of our ICSS FIRGs as compared to both the BLW (1988), and the KK (1983) samples in the $\log L_{\text {TOT }}$ versus $\log L_{\text {FIR }}$ plane.

For typical emission-line nebulae, the total luminosity ( $L_{\text {lines }}$ - summed over all emission lines) is 15-20 times what we have called $L_{\text {Tот }}(\mathrm{H} \alpha+[\mathrm{N}$ II $])$. Thus $L_{\text {lines }}$ for our ICSS FIRGs $\sim 1 \%$ of $L_{\text {FIR }}$. If the extinctions we derive for the nuclei (typically $1.5-2.0 \mathrm{mag}$ at $\mathrm{H} \alpha$ ) pertain to the entire nebulae, then the typical extinction-corrected value of $L_{\text {lines }}$ for our ICSS FIRGs $\sim 5 \%$ of $L_{\text {FIR }}$.

We have also listed in Tables 1 and 2 the nuclear
$\mathrm{H} \alpha+[\mathrm{N}$ II $]$ luminosity $\left(L_{\mathrm{NUC}}\right)$. This was measured using a box $2 \mathrm{kpc}$ on a side centered on the emission-line nucleus. Where no clear $\mathrm{H} \alpha$ nucleus is visible, we have used instead the $R$ band nucleus as the center. We find $39.93 \leq L_{\mathrm{NUC}} \leq 42.30$ for the ICSS FIRGs, with a mean value of $\log L_{\mathrm{NUC}}=41.22(\sigma=0.50)$. The distribution of $\log L_{\mathrm{NUC}}$ is shown in Figure 5. Note that we have added together the $L_{\mathrm{NUC}}$ values for systems with multiple nuclei to obtain the mean and the distribution of Figure 5. Since most of the multiple nuclei systems have a single dominant nucleus in $\mathrm{H} \alpha+[\mathrm{N}$ II $]$ (see below), we have chosen to add the nuclear luminosities together for each pair in all of the statistical investigations to follow. While this may tend to

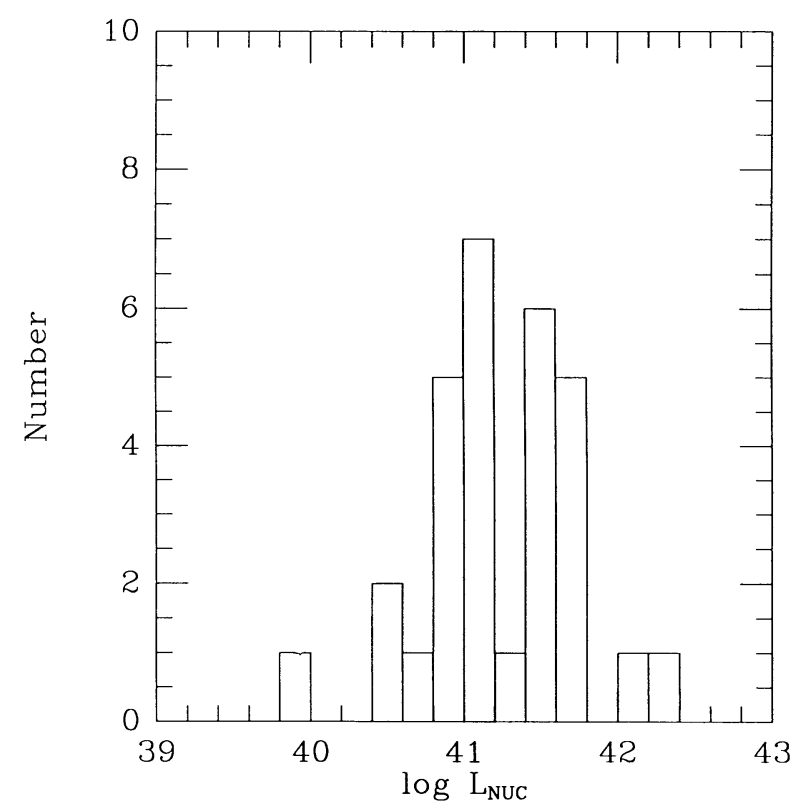

Fig. 5. - The distribution of the $\log$ of the nuclear (defined in text) $\mathrm{H} \alpha+[\mathrm{N} \mathrm{II}]$ luminosity $\left(L_{\mathrm{NUd}}\right.$, uncorrected for internal extinction, for the ICSS FIRGs. 


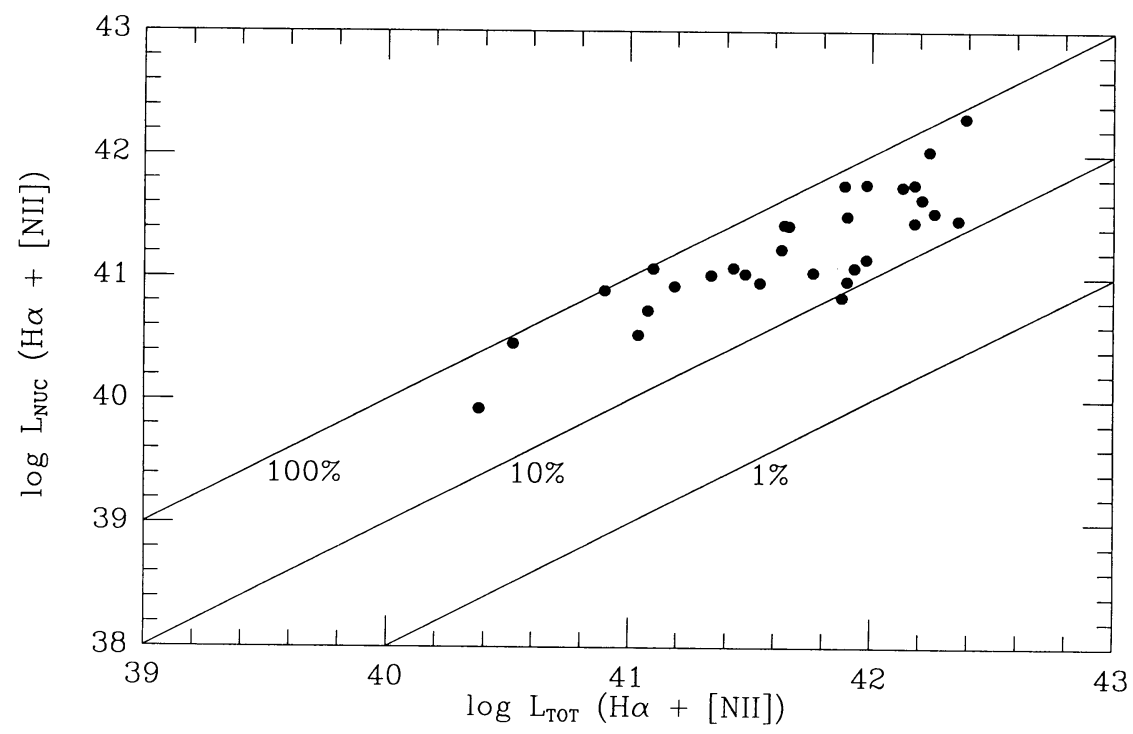

FIG. 6.-Plot of the total $\mathrm{H} \alpha+[\mathrm{N}$ II $]$ luminosity $\left(L_{\text {TOT }}\right)$ against the nuclear $\mathrm{H} \alpha+[\mathrm{N}$ II $]$ luminosity $\left(L_{\mathrm{Nud}}\right)$ of the ICSS FIRGs. The solid diagonal lines represent select ratios of nuclear-to-total $\mathrm{H} \alpha+[\mathrm{N} \mathrm{II}]$ luminosity. Both luminosities are uncorrected for internal extinction.

weight a few of the multiple nuclei systems toward higher $L_{\mathrm{NUC}}$ (e.g., NGC 3690), we feel it is the most straightforward way to compare the $2 \mathrm{kpc}$ fluxes of these systems to the more numerous single nuclei systems. Note that it is the individual nuclear fluxes and luminosities that are presented in Tables 1 and 2.

The 10 IR-bright non-ICSS galaxies have a mean log $L_{\mathrm{NUC}}=40.67(\sigma=0.68)$ and therefore have nuclei that are fainter by a factor of $\sim 3.6$ on average $(>99.5 \%$ confidence level) than the ICSS FIRGs.

To graphically depict how much of the total $\mathrm{H} \alpha+[\mathrm{N}$ II $]$ luminosity comes from within this $2 \mathrm{kpc}$ aperture, we have plotted $L_{\text {TOT }}$ versus $L_{\text {NUC }}$ in Figure 6. From this figure we can see that all the ICSS FIRGs (with the exception of $12112+0305)$ have at least $10 \%$ of their total $\mathrm{H} \alpha+[\mathrm{N}$ II $]$ fluxes arising from within our $2 \mathrm{kpc}$ aperture. However, the majority of the emission-line flux from a typical FIRG (mean extranuclear $\mathrm{H} \alpha+[\mathrm{N}$ II $]$ flux $\sim 65 \%$ of $L_{\text {TOT }}$ ) comes from regions more than $1 \mathrm{kpc}$ from the nucleus. This is consistent with our mean $\mathrm{H}+[\mathrm{N}$ II $]$ half-light radius, $r_{e} \sim 1.3 \mathrm{kpc}$, given above.

Figure $7 a$ displays the distribution of the absolute $R$ magnitude, $M_{R}$, for our IR color-selected galaxies. The mean value of $M_{R}=-21.69(\sigma=0.92)$, with a range of $-23.11 \leq M_{R} \leq-19.15$. For comparison, an $L^{*}$ galaxy has an $M_{R} \sim-21.0$ for an $H_{0}=75 \mathrm{~km} \mathrm{~s}^{-1} \mathrm{Mpc}^{-1}$ (Schecter 1976) and using a $(B-R) \sim 1.3$ (Coleman, Wu, and Weedman 1980). The brightest galaxy in the continuum is Mrk 231, with an $M_{R}=-23.11$. Mrk 231 is known to be a highly luminous type 1 Seyfert galaxy, or low-redshift QSO (see Adams and Weedman 1972; Sanders et al. 1987). The distribution of nuclear absolute $R$ magnitude, $M_{R}$ (nuc), is shown in Figure $7 b$. The mean value of $M_{R}($ nuc $)=-19.75(\sigma=0.86)$, where we have combined the nuclear $R$ band fluxes for multiple-nuclei systems to be consistent with the procedure applied to the emission-line fluxes. Here, Mrk 231 is the brightest by far of all the ICSS FIRGs, with an $M_{R}$ (nuc) $=-22.38$. The brightest single-nucleus galaxy besides Mrk 231 is $07205+7842$ with an $M_{R}($ nuc $)=-20.81$.
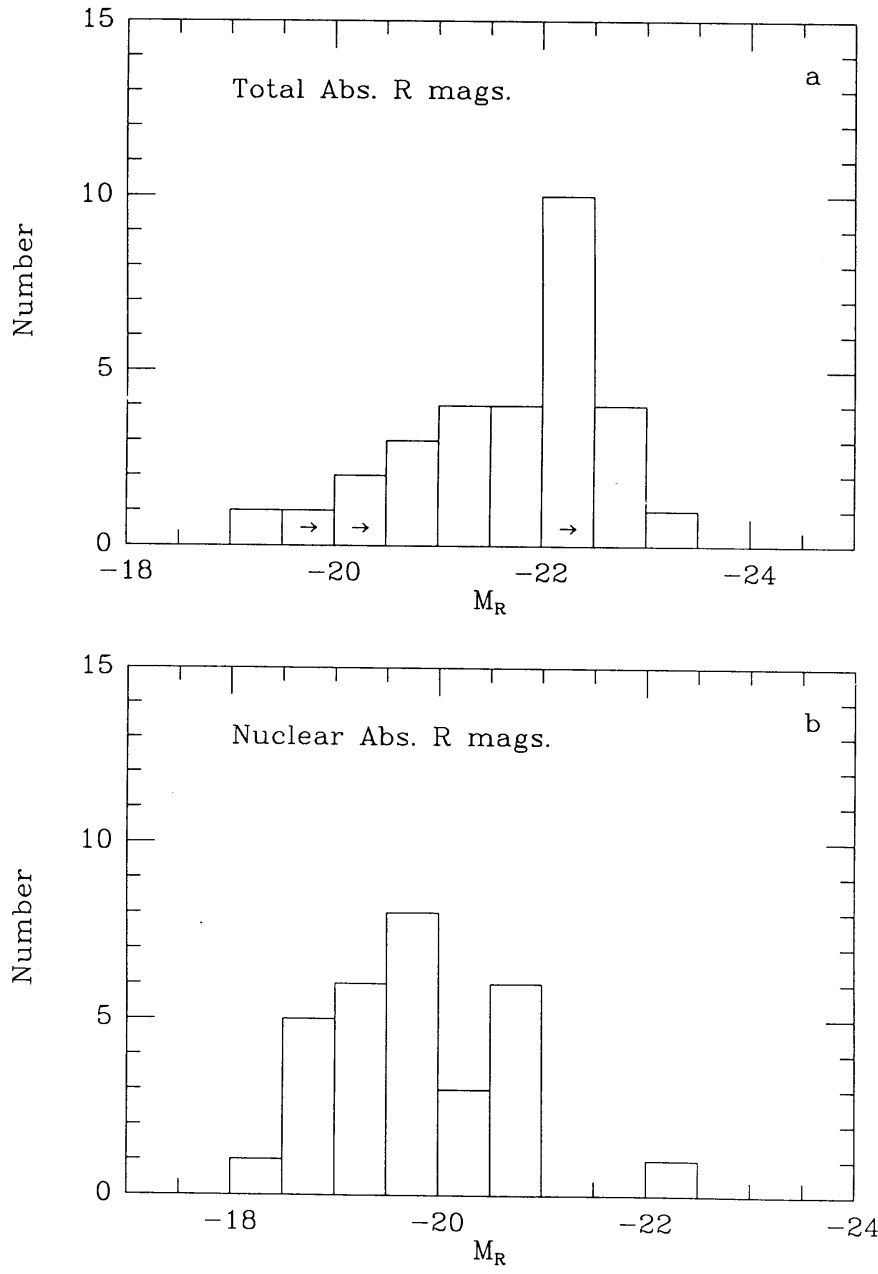

FIG. 7.-The distributions of the total and nuclear (defined in text) absolute $R$-band magnitudes, uncorrected for internal extinction, of the ICSS FIRGs. The arrows in Fig. $7 a$ indicate lower limits to the total $R$-band luminosity. 


\section{d) Individual Objects \\ i) $01217+0122,15250+3609$}

The $R$ band images of these galaxies both consist of a pair of galaxies that are apparently interacting. The bright galaxy to the east that dominates the $R$ band image of $01217+0122$, however, is very faint in the corresponding $\mathrm{H} \alpha+[\mathrm{N} \mathrm{II}]$ image of the pair. Spectra of this system reveal that the eastern galaxy is not at the same redshift as the emission-line object to the west and is most likely a foreground object. The $\mathrm{H} \alpha+[\mathrm{N}$ II $]$ image is therefore slightly undersubtracted (our spectra reveal no $\mathrm{H} \alpha+[\mathrm{N}$ II $]$ emission from this galaxy). The fluxes and luminosities presented in Table 1 pertain only to the western stellar-appearing galaxy.

The small galaxy to the north in the continuum image of $15250+3609$ similarly vanishes in the emission-line image. We do not have a spectrum of this object, however, and we cannot calculate its redshift directly.

\section{ii) NGC 1222 (IRAS 03064-0308, Mrk 603)}

The $R$ band continuum image of this galaxy consists of one dominant nucleus, and three to four secondary peaks to the east and southwest. There appears to be a great deal of patchy obscuration around the nucleus, with evidence for one or two dust lanes possibly crossing each other at right angles over the inner $\sim 0.7 \mathrm{kpc}$. At low surface brightness levels, the continuum appears elliptical, with an orientation along a P.A. $\sim 150^{\circ}$

In the light of $\mathrm{H} \alpha+[\mathrm{N}$ II $]$, the nucleus shows up clearly, as do four other hot spots (most likely giant $\mathrm{H}$ II regions) with sizes of $0.5-1.0 \mathrm{kpc}$. The brightest of these to the southwest is $\sim 3 \mathrm{kpc}$ from the nucleus and in fact may be the stripped nucleus of the galaxy with which the main body of NGC 1222 is merging. The $\mathrm{H} \alpha+[\mathrm{N}$ II $]$ luminosity of this hot spot is $\sim 4 \times 10^{40} \mathrm{ergs} \mathrm{s}^{-1}$ ( $\sim 7 \%$ of the nucleus). At the highest levels the emission-line nebula is oriented at a P.A. $\sim 80^{\circ}$, roughly perpendicular to the large-scale continuum image. Wispy, filamentary structure can also be seen at radii of $\sim 3-4 \mathrm{kpc}$, with a maximum extent of $\sim 8-9 \mathrm{kpc}$.

iii) NGC 1614 (IRAS 04315-0840, Arp 186, Mrk 617)

The circumnuclear region (inner few $\mathrm{kpc}$ ) of the continuum image of this galaxy is composed of a single nucleus and radiating spiral arms of unequal brightness. The isophotes twist through $\sim 90^{\circ}$ as one moves to lower surface brightness levels. The faintest features are truly the most spectacular, comprising (1) a linear "tail" to the southwest that is $\leq 8 \mathrm{kpc}$ wide and extends almost $20 \mathrm{kpc}$ from the nucleus, and (2) a large curving arc to the east that reaches $\sim 11 \mathrm{kpc}$ from the nucleus at its maximum.

In the $\mathrm{H} \alpha+[\mathrm{N}$ II $]$ image we can see a nuclear peak, which is coincident with the broad-band nucleus, as well as the inner spiral arms. The upper (eastward) arm appears brighter in $\mathrm{H} \alpha+[\mathrm{N} I \mathrm{I}]$, however, contrary to what we see in the continuum image. There also appear to be $\mathrm{H}$ II region complexes (with diameters of $1-1.5 \mathrm{kpc}$ ) strung out along the "tail" feature at distances of up to $10 \mathrm{kpc}$ from the nucleus, as well as some faint emission coincident with the eastern arc.

iv) NGC 2623 (IRAS 08354 +2555, Arp 243)

The bizarre morphology of this galaxy was noticed early by Toomre and Toomre (1972), who suggested it as being the result of a nearly completed merger of two spiral galaxies of similar size. In our broad-band $R$ image, we are able to see what are apparently two tidal tails oriented along a P.A. $\sim 50^{\circ}$.
Each of these tails extends almost $25 \mathrm{kpc}$ from the dusty nucleus. A vestigial spiral structure is also evident extending over the inner $10 \mathrm{kpc}$, along a P.A. $\sim 0^{\circ}$. These arms may attach to the nucleus via a bar that runs E-W.

In the light of $\mathrm{H} \alpha+[\mathrm{N}$ II $]$ the nucleus dominates the emission, but two or three hot spots are visible to the south. Two of these lie along one of the inner spiral arms mentioned above, but one (the brightest) does not seem coincident with this continuum structure. The spectacular tidal tails are not visible in the narrow-band image, except for some faint emission along the NE tail. Thus, the tidal tails are predominantly seen only in starlight, with the emission-line gas confined to the circumnuclear ( $\sim 4 \mathrm{kpc}$ radius) region.

$$
\text { v) } 10565+2448
$$

The $R$ band image of $10565+2448$ is made up of two disk galaxies in the midst of a quite damaging encounter, with the two nuclei separated by $\sim 20 \mathrm{kpc}$. Two spiral arms are visible in the western galaxy, oriented along a P.A. $\sim 110^{\circ}$. The eastern arm actually curves all the way around in a large loop, reaching a maximum distance from the nucleus of $\sim 10 \mathrm{kpc}$. In addition to the nucleus itself, we can see two other continuum peaks in the western galaxy, the brighter one being approximately $6 \mathrm{kpc}$ to the east, and the fainter one at $\sim 9.5 \mathrm{kpc}$ to the south. A bridge of continuum emission stretches between the two nuclei, eventually curving back toward the western galaxy in an arc.

In light of the $\mathrm{H} \alpha+[\mathrm{N}$ II $]$ emission lines, this system is much less spectacular. Only the nuclei are visible, with the western galaxy being almost a factor of 17 more luminous (log $L_{\mathrm{NUC}}=41.40$ ) than its eastern counterpart. There may be some weak $\mathrm{H} \alpha+[\mathrm{N}$ II $]$ emission along the upper (eastern) spiral arm of the bright galaxy. The tail and bridge of luminous material so prominent in the continuum image are not radiating in emission lines, at least not at a level of $10^{-16}$ ergs $\mathrm{cm}^{-2} \mathrm{~s}^{-1} \operatorname{arcsec}^{-2}$.

vi) NGC $3690($ IRAS 11257+5850, Arp 299, Mrk 171)

The peculiar morphology of this galaxy was first noticed by Arp (1966), but it was misidentified as number 296 in his Table 1 . The severely distorted system actually consists of two identified galaxies: IC 694 to the east, and NGC 3690 to the west. For our purposes we will call both galaxies the NGC 3690 system. Previous imaging by Gehrz, Sramek, and Weedman (1983), and Joy et al. (1989) has revealed three prominent peaks in the mid-IR, which are labeled as Gehrz A, Gehrz B, and Gehrz $\mathrm{C}$ in Figure 1. These peaks are also discernible in the broad-band images of Friedman et al. (1987), and the $6 \mathrm{~cm}$ and $20 \mathrm{~cm}$ radio maps of Gehrz, Sramek, and Weedman (1983). Only two peaks are seen in the near-IR maps of this system by Telesco, Decher, and Gatley (1985), corresponding to Gehrz A and Gehrz B in Figure 1, but they indicate that a shoulder in the near-IR emission may be present at the position of Gehrz C. Since much of the near-IR $(2 \mu \mathrm{m})$ flux presumably comes from red giants, Telesco, Decher, and Gatley (1985) argue that the peaks seen at the positions of Gehrz A and Gehrz B are in fact the true nuclei (centers of mass) of IC 694 and NGC 3690, respectively. The far-infrared profiles of Joy et al. (1989) indicate that $60 \%$ of the far-infrared emission from the system is originating in an unresolved source whose location is consistent with the position of Gehrz A. The remaining $40 \%$ of the system's far-infrared emission is coming from either a single compact source, or a pair of marginally resolved sources at the location of Gehrz B, and Gehrz C. 
Besides the multiple-peaked continuum structure of the NGC 3690 system seen at the highest surface brightness levels of Figure 1, we also see a highly irregular, patchy internuclear morphology made up of many loops, or arcs of varying sizes. At the lowest surface brightness levels we can make out largescale features that appear to be tails, or streamers. One of these extends $\sim 8 \mathrm{kpc}$ to the east from IC 694 , while another extends nearly $12 \mathrm{kpc}$ to the NW from NGC 3690 (this linear feature is made up of a string of condensations, or knots). A faint, wispy, curving tail is also visible to the south of IC 694.

The $\mathrm{H} \alpha+[\mathrm{N}$ II $]$ image of the NGC 3690 system is even more complicated than the continuum image described above. The body of IC 694 breaks up into at least four hot spots at the $5 \times 10^{-14}$ ergs $\mathrm{cm}^{-2} \mathrm{~s}^{-1} \operatorname{arcsec}^{-2}$ level. The body of NGC 3690 itself, has two $\mathrm{H} \alpha+[\mathrm{N}$ II $]$ peaks above the $10^{-13}$ ergs $\mathrm{cm}^{-2} \mathrm{~s}^{-1} \operatorname{arcsec}^{-2}$ level. Gehrz, Sramek, and Weedman (1983) locate two $\mathrm{H} \alpha$ emission-line peaks at the positions of $B$ and $C$. Very weak emission was detected from the position of A. Using a long slit oriented at a P.A. $\sim 85^{\circ}$ going through the position of A, Friedman et al. (1987) found $\mathrm{H} \alpha$ emission extending over several kpc, with peaks arising in a number of small condensations (of diameter $\leq 500 \mathrm{pc}$ ) in the central regions of IC 694 and NGC 3690. Since determination of the nuclei of these galaxies is difficult in the emission-line image, we have employed the results of the previous work cited above, and measured the nuclear $\mathrm{H} \alpha+[\mathrm{N}$ II $]$ emission-line flux in 2 $\mathrm{kpc} \times 2 \mathrm{kpc}$ boxes, centered at three locations in the emissionline contour plot of Figure 1 . These correspond roughly to the positions of the Gehrz nuclei labeled C, A, and B, respectively. At the lowest surface brightness levels, the NGC 3690 system exhibits a magnificent large-scale, filamentary structure made up of arcs and loops that surround the galaxies at radii $\sim 6-12$ kpc. The largest of these are just visible in the contour plots to the east and SE of IC 694. In the gray-scale image of the emission-line nebula in Figure $8 a$ (Plate 7), we have overexposed the nuclear region to make the faint, large-scale structure more clearly visible. The complex nature of the nuclei of these galaxies is evidently reflected by the chaotic emission-line nebula that, at the lowest signal-to-noise levels, seems to fill our entire $2^{\prime} .5 \times 22^{\prime} .5$ field of view! The large angular size and intricate morphology of the emission-line nebula in NGC 3690 make it one of the most spectacular galaxies in our IR colorselected sample.

\section{vii) $12018-1814(\operatorname{Arp} 244)$}

This system has one of the strangest $R$ band morphologies of any of the color-selected FIRGs. It is apparently composed of two interacting galaxies, whose nuclei are separated by $\sim 18$ kpc. Although the interaction has been quite damaging, the southern galaxy has retained a distorted spiral structure, with arms extending along a P.A. $\sim 145^{\circ}$. The northern galaxy, on the other hand, displays more of a head/tail structure. Also visible in the continuum image is a fanlike structure that originates in the southern galaxy and extends for $\sim 21 \mathrm{kpc}$ to the SW.

The nucleus of the northern galaxy is clearly the brightest object in the $\mathrm{H} \alpha+\left[\mathrm{N}_{\text {II }}\right]$ image of $12018-1814$. With an $L_{\mathrm{NUC}}=2.7 \times 10^{41} \mathrm{ergs} \mathrm{s}^{-1}$, it is almost 12 times as luminous as the nucleus of the southern galaxy. In 12018-1814 the ionized gas traces the continuum very well, except for the absence of line emission at the location of the fanlike structure mentioned above. We also see a shoulder, or jet, of $\mathrm{H} \alpha+[\mathrm{N} \mathrm{II}]$ emission coming off of the nucleus of the northern galaxy at a
P.A. $\sim 10^{\circ}$. This is visible in the continuum image as well. One emission-line peak can also be seen between the two nuclei, roughly at the position of the end of one of the southern galaxy's spiral arms.

$$
\text { viii) NGC } 4194 \text { (IRAS 12116+5448, Arp 160) }
$$

The broad-band image of this galaxy has two very interesting structures. The first of these is a relatively sharp edge to the continuum light $\sim 2 \mathrm{kpc}$ southeast of the nucleus. The second is a spur, or jet ( $\sim 1 \mathrm{kpc}$ long), just east of the nucleus pointing to the north.

In the $\mathrm{H} \alpha+[\mathrm{N}$ II $]$ image a faint halo of emission $(\sim 3 \mathrm{kpc}$ in diameter) surrounds a single dominant nucleus that aligns with the peak in the continuum. There are also three circumnuclear hot spots, the brightest of which is $\sim 600 \mathrm{pc}$ from the nucleus to the northeast. At low surface brightness levels, two $\mathrm{H} \alpha+[\mathrm{N}$ II $]$ tails are apparent, one curving to the east, and the other curving to the south. In Figure $8 b$ (Plate 8), we present a gray-scale image of the $\mathrm{H} \alpha+[\mathrm{N}$ II $]$ nebula of NGC 4194, in which we have overexposed the nucleus to make these tails more apparent. The edge-brightened, looplike structure of the southern brighter tail can be seen in this image. The eastern tail may possess a similar morphology, but its faintness makes such an identification difficult.

$$
\text { ix) } 13136+6223(\operatorname{Arp} 238)
$$

The complex $R$ band morphology of this system can easily be understood as arising from the interaction of two spiral galaxies of nearly equal size. The inner spiral arms of both galaxies, while distorted, are still visible. The nuclei are separated by $\sim 21 \mathrm{kpc}$ and are connected by a bridge of luminous material. Two opposing tidal tails emerge from the galaxies at a P.A. $\sim 120^{\circ}$. The southeast tail continues off the edge of the CCD field, but the other extends $\sim 16 \mathrm{kpc}$ from the western nucleus before curving to the northeast. We also see a small spur coming from the circumnuclear region of the eastern galaxy and pointing in a southerly direction. This could be another tidal tail generated as a result of the interaction.

In the narrow-band image of $13136+6223$, the eastern galaxy is clearly brighter with an $L_{\mathrm{NUC}}=5 \times 10^{41}$ ergs s $^{-1}$ (almost a factor of 10 greater than the $L_{\mathrm{NUC}}$ of the western galaxy). The emission-line contours of this galaxy are almost perfectly circular. Most of the connecting bridge seen in the broad-band image does not show up in emission lines, but the northwest tail with a hot spot at the location that the tail curves to the northeast is clearly visible. The elongated structure of the higher contour levels in the western galaxy are not at the same P.A. as the spiral arm visible in the broad-band image. This $\mathrm{H} \alpha+[\mathrm{N}$ II $]$ emission instead lies along the vector connecting the two nuclei.

$$
\text { x) } M r k 273(\text { IRAS } 13428+5608)
$$

Markarian (1969) commented that this interesting galaxy had a "large straight protrusion." Wehinger and Wyckoff (1977) using broad-band filters centered at $\sim 5000 \AA$, noted this peculiar linear feature extending to the south, as well as an elongated, possibly double nucleus, and faint curved wisps to the northeast. Adams (1977) again using broad-band green filters noted an irregular inner structure plus a long straight "plume" to the south which did not align with the nucleus. Faint wisps were also seen to the east of the nucleus. The most obvious feature in our broad-band $R$ image is the narrow, straight tail extending $\sim 40 \mathrm{kpc}$ from the nucleus. The ratio of the length of the tail to its width is $\sim 7: 1$. Also visible is a 
"spur" that reaches to the southeast from a position that is about one-fourth of the way along the tail. Faint diffuse emission extends from the nucleus $\sim 23 \mathrm{kpc}$ to the northeast.

In the narrow-band $\mathrm{H} \alpha+[\mathrm{N}$ II] image of Mrk 273, the linear tail is not present. It is instead replaced by patchy areas of ionized gas that extend along the same P.A. $\left(\sim 180^{\circ}\right)$, with similar emission to the northeast coincident with the wispy continuum light described above. This extranuclear $\mathrm{H} \alpha+[\mathrm{N} \mathrm{II}]$ emission is in the form of arcs or loops. The nuclear region is triangular, with two prominent peaks aligned along a P.A. $\sim 55^{\circ}$. These peaks are separated by less than 2 $\mathrm{kpc}$, and thus we have treated them as a single nucleus in the calculation of $L_{\mathrm{NUC}}$.

\section{xi) $15163+4255($ Mrk 848)}

The continuum image of $15163+4255$ consists of what appears to be two galaxies in the process of merging, whose nuclei are separated by $4-5 \mathrm{kpc}$. The northern nucleus has a peak surface brightness almost a factor of 4 greater than the southern nucleus, but the latter appears to have a dust lane bisecting it at a P.A. $\sim 155^{\circ}$. Two opposing tidal tails, along a P.A. $\sim 160^{\circ}$, curve back toward, and actually pass behind, the nuclei.

In the light of $\mathrm{H} \alpha+[\mathrm{N}$ II], the two nuclei are very conspicuous, with the northern one having an emission-line luminosity of $4.7 \times 10^{41}$ ergs $\mathrm{s}^{-1}$ (a factor of $\sim 6$ greater than the emission-line luminosity of the southern nucleus). The opposing tidal tails are still visible in emission lines, but they are faint (at levels of $1-2 \times 10^{-16} \mathrm{ergs} \mathrm{cm}^{-2} \mathrm{~s}^{-1} \operatorname{arcsec}^{-2}$ ), curving around to end in two hot spots that are approximately $9 \mathrm{kpc}$ to the west and $11 \mathrm{kpc}$ to the south of the northern and southern nuclei, respectively.

\section{xii) NGC 660, NGC 2146, NGC 3079, and NGC 5900}

The powerful far-infrared (non-ICSS) galaxies NGC 660 NGC 2146, NGC 3079, and NGC 5900 all exhibit relatively similar broad-band continuum morphologies, along with possessing quite interesting and related narrow-band $\mathrm{H} \alpha+[\mathrm{N}$ II $]$ features. We will, therefore, discuss these four galaxies together. NGC 660, NGC 2146, NGC 3079, and NGC 5900 are all nearly edge-on spiral galaxies. The first three have a rather patchy appearance in $R$, plus a prominent dust lane that runs along the major axis of their disks. NGC 660 may also have a second dust lane in its NE quadrant which makes an angle of $\sim 30^{\circ}$ with the major axis. NGC 5900 has a major axis dust lane running the length of its disk, but overall it appears much smoother in the continuum than NGC 660, NGC 2146, or NGC 3079. There also may be a faint tail extending toward the southeast $\sim 5 \mathrm{kpc}$ beyond the disk of NGC 5900 .

The narrow-band images of NGC 660, NGC 2146, and NGC 3079 are dominated by nuclear $\mathrm{H} \alpha+[\mathrm{N} \mathrm{II}]$ emission, as well as line emission from the many giant $\mathrm{H}$ in regions scattered throughout their disks. In addition, in NGC 660 (see Fig. 8c [Plate 9]) there are two faint jets which may be the sides of a loop or bubble, extending $\sim 2 \mathrm{kpc}$ out from the nucleus at a P.A. $-40^{\circ}$ (along the minor axis). In NGC 3079, an almost completely closed loop of $\mathrm{H} \alpha+[\mathrm{N}$ II $]$ emission about $1.5 \mathrm{kpc}$ in diameter is visible emerging from the nucleus at a P.A. $\sim 65^{\circ}$. This feature was first noticed by Ford et al. (1986), who remarked on its coincidence with the radio loop reported by Duric and Seaquist (1986). Ford et al. (1986) argue that both the optical and radio features are caused by the interaction of plasma, ejected by the active nucleus, with the ISM. At the lowest surface brightness levels in our narrow-band image of
NGC 3079, we can also see what appears to be an “ $X$ " pattern of radiating filaments with lengths of between 2 and $6 \mathrm{kpc}$, roughly centered on the nucleus. The gray-scale image of NGC 3079 presented in Figure $8 d$ (Plate 10) shows these very faint filaments emerging from the disk of the galaxy. At least two, and possibly three, of these filaments can be seen to the east of the disk, with one to the southwest, and the faintest of the group extending to the northwest. The loop of emission-line gas noted above is overexposed in this image of NGC 3079 but is visible in the contour plot of Figure 1. In the gray-scale $\mathrm{H} \alpha+[\mathrm{N}$ II $]$ image of NGC 2146 we can see a more chaotic system of filaments than in NGC 3079, emerging to the northeast of the prominent dust lane. Here, the many filaments are confined to the circumnuclear region of NGC 2146, and it is unclear whether or not they actually exist above the plane (along the rotation axis) of the disk. In appearance they resemble the filaments and loops seen in the narrow-band images of M82 (McCarthy, Heckman and van Bruegel 1987), much more than they do the seemingly aligned structure visible in NGC 3079. Nonetheless, as with NGC 660, NGC 3079, and NGC 5900 (see below), this intricate filamentary or bubble-like structure may have a direct causal link to the energetic phenomena that are apparently occurring in the nuclei of these galaxies.

In the $\mathrm{H} \alpha+[\mathrm{N}$ II] image of NGC 5900, no $\mathrm{H}$ II regions are visible. Two peaks in the emission can be seen along the major axis of the disk, one centered on the $R$ band peak, and the other $\sim 2 \mathrm{kpc}$ to the northwest. There is also a faint jetlike feature coming from the latter peak along a P.A. $\sim-145^{\circ}$, almost perpendicular to the disk major axis, with a length $\sim 5$ kpc. A similar linear emission feature may be visible directly opposite the disk, extending $\sim 3 \mathrm{kpc}$ along a P.A. $\sim 35^{\circ}$. The faint tail noted in the continuum image is seen in $\mathrm{H} \alpha+[\mathrm{N} \mathrm{II}]$ emission as well, although it cannot be seen in the contour plot of Figure 1, extending for $\sim 4 \mathrm{kpc}$ beyond the disk (as defined by our narrow-band image) out along a P.A. $\sim 130^{\circ}$.

\section{xiii) $M r k 266$}

The twin nucleus, merging galaxy Mrk 266 has been the focus of a great deal of intense study at many wavelengths. Petrosian (1980) published an early broad-band contour plot, noting the two nuclei, and a "superassociation" some 20 " to the SW. A more detailed optical and radio $(21 \mathrm{~cm})$ study of this galaxy has recently been published by Hutchings, Neff, and van Gorkom (1988, hereafter HNG), in which they note, referring to their broad-band $B$ and $R$ images, much "faint extended structure that is complex and highly suggestive of tidal tails." The $R$ band continuum image we present as a contour plot in Figure 1 shows much of the greatly distorted morphology noted previously. Among the interesting features are the tails or loops to the SE, and the very faint "plume" to the NW. The brighter of the two nuclei, to the north, shows very symmetrical contours, while the fainter nucleus displays a quite strange "two-pronged" morphology that points toward the southwest. A dust lane may cut across between the nuclei at a P.A. $\sim 120^{\circ}$.

In the light of $\mathrm{H} \alpha+[\mathrm{N}$ II $]$, Mrk 266 is the most spectacular of the non-ICSS galaxies. A complex filamentary structure extends for almost $27 \mathrm{kpc} \mathrm{N}-\mathrm{S}$ and almost $31 \mathrm{kpc} \mathrm{E}-\mathrm{W}$. There is one prominent nucleus, which aligns with the brightest $R$ band nucleus, and a fainter (by about a factor of 1.5) peak that aligns with the other $R$ band nucleus. There is a long curving tail that extends north $\sim 20 \mathrm{kpc}$ from the vicinity of the southern nucleus, with an edge-brightened morphology that gives it the appearance of being a long, stretched-out loop. The 
positive gray-scale $\mathrm{H} \alpha+[\mathrm{N}$ II $]$ image displayed in Figure $8 e$ (Plate 11) not only shows this loop very clearly, but also two other large-scale loops to the east. At low surface brightness levels, there is a large, fanlike structure that extends $\sim 11 \mathrm{kpc}$ to the southwest from the fainter nucleus, which, upon closer inspection, is made up of many loops, arcs, or bubbles. The extent and complex nature of this low-level emission can be seen in the negative gray-scale $\mathrm{H} \alpha+[\mathrm{N} \mathrm{II}]$ image of Figure $8 f$ (Plate 12). At higher surface brightness levels, we can see what appears to be an arm coming from the southern nucleus and reaching first to the east, and then to the south, before disappearing in the frothy, filamentary nebulosity $\sim 6 \mathrm{kpc}$ away. This arm is clearly visible in the positive gray-scale image of Figure $8 e$. A long $(\sim 16 \mathrm{kpc})$ fairly linear structure is also visible originating near the brighter nucleus, along a P.A. $\sim 125^{\circ}$. This feature can be seen in Figure $8 f$ extending out from the two eastern loops mentioned above.

HNG show an image of Mrk 266 in the light of the [O III] $\lambda 5007$ line which displays many of the basic features noted above, although much of the faint off-nuclear emission that can be seen in our Figure 1 is not visible in their contour plot. Their $\mathrm{H} \alpha$ contour shows none of the detailed structure that is seen in our narrow-band image. The two nuclei and some of the circumnuclear emission is seen in their Figure 2, but the extended, filamentary nature of the nebulae is not apparent. Our $\mathrm{H} \alpha+[\mathrm{N}$ II $]$ image more closely resembles the [O III] plot of HNG, suggesting the morphology of Mrk 266 is in fact similar in both emission lines.

\section{DISCUSSION}

\section{a) Ionization of the Emission-Line Nebulae}

It is evident from Figure 1, and the comments of $\S$ III, that large (galaxy-scale) emission-line nebulae with $\mathrm{H} \alpha$ luminosities comparable to those found in the brightest Sc galaxies are common in our sample of ICSS FIRGs. A fundamental question concerns the source of this $\mathrm{H} \alpha$ emission. Are these nebulae made up of many giant $\mathrm{H}$ II regions, as in a typical spiral galaxy, or are they of a different nature entirely? Clearly some of the IR color-selected galaxies contain off-nuclear $\mathrm{H} \alpha$ surface brightness peaks that resemble $\mathbf{H}$ II regions. Others display large-scale filamentary structures that look nothing like the $H$ II region complexes of normal spiral galaxies. Still other ICSS FIRGs, however, have extended, $\mathrm{H} \alpha$ nebulosities that appear "smooth" down to the lowest isophotal levels plotted in Figure 1. By "smooth," we mean that the nebulae have no local maxima or coherent structure, showing only a gradual falloff in surface brightness from the nucleus. To find out whether these smooth nebulae could in fact be made up of a large number of blended $\mathrm{H}$ II regions, we can examine the $\mathrm{H} \alpha+[\mathrm{N} \mathrm{II}]$ structure of two of the nearby, well-resolved spiral galaxies that are not members of the IR color-selected sample.

The galaxies, NGC $1667(z=0.015)$ and NGC 4536 $(z=0.006)$, are both relatively close spirals that have $\mathrm{H} \alpha+[\mathrm{N}$ II $]$ luminosities comparable to our IR color-selected galaxies. More importantly, they exhibit the classical H II region, dotted spiral arm morphology mentioned above. We can use a median filter on the emission-line images of these galaxies to simulate their appearance at a $z=0.05$ and a $z=0.10$, assuming a resolution of 1.15 (the typical seeing during our observations). Essentially, we are smoothing the images using a box with the same area as a circle of diameter equal to the number of parsecs corresponding to $1^{\prime \prime} .5$ at a red- shift of $z=0.05$ and $z=0.10$. When we perform this smoothing, we find that the $\mathrm{H}$ II regions that are prominent in the original images are easily discernible at a simulated redshift $z=0.05$, but only the largest conglomerations are visible by a redshift of $z=0.10$. We should, therefore, be able to see individual $\mathrm{H}$ II region complexes in all the ICSS FIRGs with a $z \leq 0.07$ if they do indeed have an $\mathrm{H} \alpha$ nebular structure similar to NGC 1667 and NGC 4536. Using this as a guide, only $\sim 25 \%$ of the ICSS FIRGs with $z \leq 0.07$ have morphologies suggesting that giant $\mathrm{H}$ II regions, like the ones we see in NGC 1667 and NGC 4536, contribute significantly to their appearance in emission lines. Also, nearly $\frac{2}{3}$ of the smooth ICSS FIRGs have $z \leq 0.07$, suggesting that extranuclear $H$ II regions are not responsible for their $\mathrm{H} \alpha$ nebulosities. On the other hand, the "smooth" $\mathrm{H} \alpha+[\mathrm{N}$ II $]$ nebulosities may be ionized by young stars which are not distributed as they are throughout the disks of normal spirals. Only a detailed analysis of the long-slit spectra (currently in progress) will be able to establish their true nature.

While only $\sim 25 \%$ of the nearby ICSS FIRGs have $\mathrm{H}$ II region complexes that dominate their extranuclear appearance, nearly $50 \%$ of the nearby FIRGs have between one and three $\mathrm{H} \alpha+\left[\begin{array}{ll}\mathrm{N} & \mathrm{II}\end{array}\right]$ "knots" visible in their continuumsubtracted images. These knots have $\mathrm{H} \alpha$ luminosities of $\sim 10^{39}-10^{41}$ ergs $\mathrm{s}^{-1}$ (uncorrected for internal extinction). They are therefore comparable to the most luminous known extragalactic H II regions (the "giant" or "supergiant" $\mathrm{H}$ II regions of Kennicutt 1984, 1988).

The nuclear spectra of a majority of the ICSS FIRGs show that the optical continuum is dominated by young, hot stars (Armus, Heckman, and Miley 1989). For the ICSS FIRGs with adequate spatial resolution and high off-nuclear signal-tonoise ratio, this was found to hold true not only in the nucleus, but also at distances of up to $4 \mathrm{kpc}$ from the nucleus. For the more distant ICSS FIRGs, the extracted nuclear spectra actually included a significant fraction of the total continuum flux as measured here by $M_{R}$ (the mean is $\sim 31 \%$ of $L_{R}$ for galaxies with $z \geq 0.05$ ). Thus, the $R$ band light is evidently dominated by the light from young stars. If these young stars are also responsible through photoionization for the optical line emission and also for the far-infrared emission, then we might expect to see a correlation of the total $R$ absolute magnitude, $M_{R}$, with $L_{\text {TOT }}$ and $L_{\text {FIR }}$. Figure 9 is a plot of $M_{R}$ versus the total $\mathrm{H} \alpha+\left[\begin{array}{ll}\mathrm{N} & \mathrm{II}] \\ \mathrm{f}\end{array}\right]$ luminosity $\left(L_{\mathrm{TOT}}\right)$, the nuclear $\mathrm{H} \alpha+[\mathrm{N} \mathrm{II}]$ luminosity $\left(L_{\mathrm{NUC}}\right)$, the ratio of the nuclear to the total $\mathrm{H} \alpha+[\mathrm{N}$ II $]$ luminosity, and the far-infrared luminosity $\left(L_{\mathrm{FIR}}\right)$, respectively. There is a correlation of $M_{R}$ with both log $L_{\text {TOT }}$ and $\log L_{\text {FIR }}$. The least-squares fit line is drawn through the data in both cases. The slope of the line in Figure $9 a$ is -0.60 , which gives us $L_{\text {TOT }} \propto L_{R}^{1.49 \pm 0.33}$. The slope of the line in Figure $9 d$ is -0.68 with $L_{\text {FIR }} \propto L_{R}^{1.71 \pm 0.46}$. Note that due to the scatter in various luminosity correlations reported throughout this paper, the slopes and power-law indices given are the averages of the values of these parameters obtained by performing the linear regression of " $y$ " on " $x$," and of " $x$ " on " $y$." The uncertainties reflect the values given by the choice of dependent and independent variable.

We can test whether the correlation in Figure $9 d$ could be a result of a selection effect caused by the absence of galaxies with moderate to high $L_{R}$, but low $L_{\text {FIR }}$. As described in Heckman, Armus, and Miley (1987), we initially chose ICSS FIRGs for broad-band imaging and spectroscopy that were optically faint as seen on the Palomar and ESO/SRC Southern 

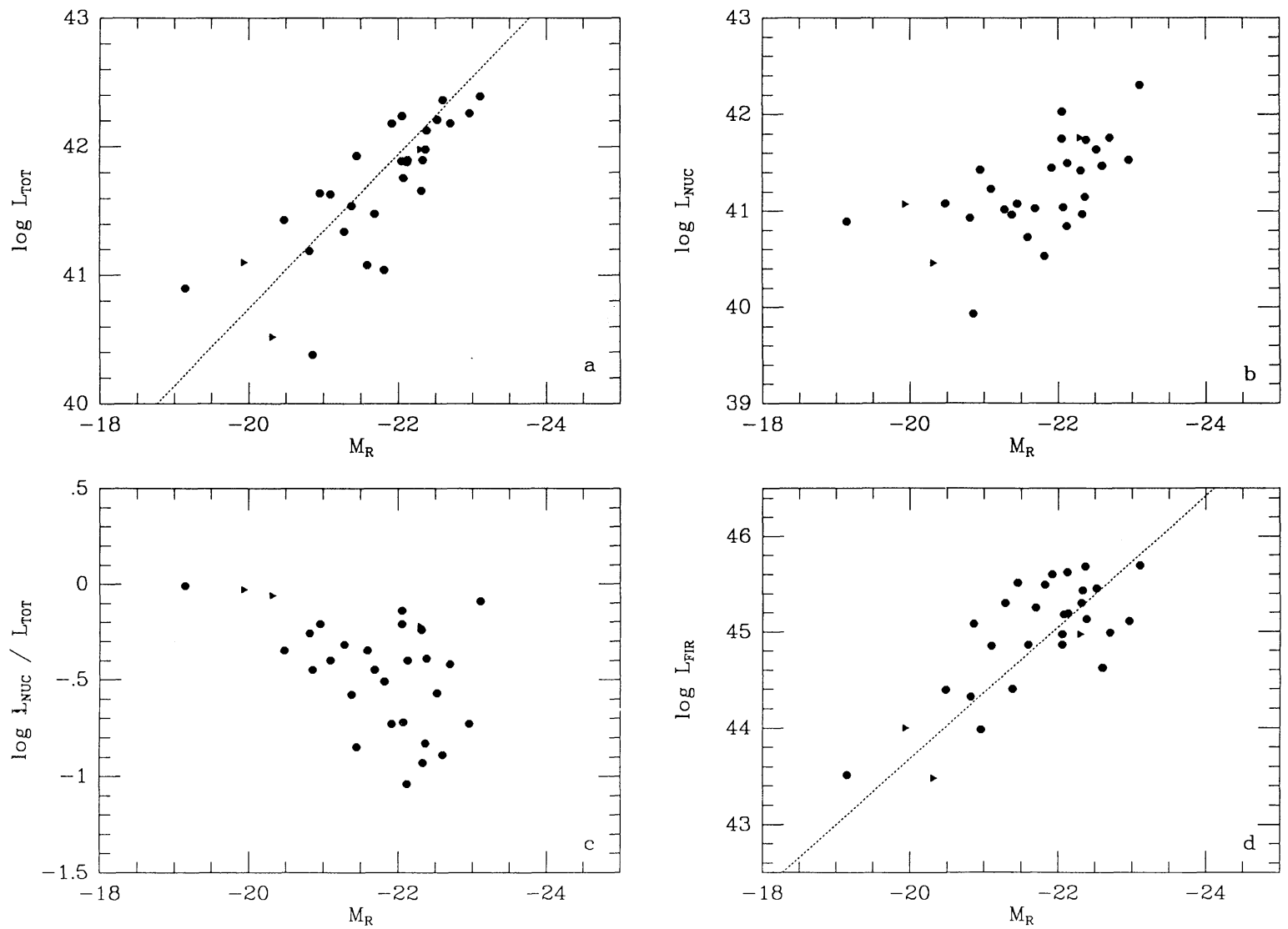

FIG. 9.-Plot of the total absolute $R$ band magnitude against (a) the total $\mathrm{H} \alpha+[\mathrm{N}$ II $]$ luminosity, (b) the nuclear $\mathrm{H} \alpha+[\mathrm{N}$ II $]$ luminosity, (c) the ratio of the nuclear-to-total $\mathrm{H} \alpha+[\mathrm{N} \mathrm{II}]$ luminosity, and $(d)$ the far-infrared luminosity of the ICSS FIRGs. The linear least-squares fit to the data is drawn as a dotted line in Figs $9 a$ and $9 d$. The arrows indicate lower limits to the $R$ band absolute magnitudes. All luminosities are uncorrected for internal extinction.

Sky Surveys in the hope of picking out the galaxies with the most extreme infrared-to-optical properties. The present sample of ICSS FIRGs overlaps with the earlier samples in the case of 10 galaxies. If we exclude these ICSS FIRGs from Figure $9 d$, then we calculate a least-squares fit to the data having a slope $=-0.67\left(L_{\text {FIR }} \propto L_{R}^{1.68 \pm 0.33}\right)$. The inclusion of the 10 galaxies chosen to be faint optically (in apparent magnitude) does not seem to be governing the correlation seen in Figure $9 d$. As a further check on the presence of a selection bias, we can look at the far-infrared flux limited sample (the 28 ICSS FIRGs with $S_{60 \mu \mathrm{m}} \geq 10$ Jy chosen without regard to optical brightness) from Armus, Heckman, and Miley (1987). We have absolute, total blue magnitudes for 27 of these 28 galaxies, and we have $R$ band images in the present paper (and thus $M_{R}$ ) for 13 members of the flux-limited sample. A plot of $M_{R}$ versus $L_{\mathrm{FIR}}$ for these 13 galaxies has a least-squares best-fit slope of -0.63 , implying that $I_{\text {FIR }} \propto L_{R}^{1.57 \pm 0.13}$. A plot of $M_{B}$ versus $L_{\mathrm{FIR}}$ for the 27 infrared flux-limited galaxies can be fitted with a line of slope $=-0.60$, implying that $L_{\text {FIR }} \propto L_{B}^{1.50 \pm 0.35}$. The absolute blue magnitudes for the flux-limited sample galaxies were derived from the apparent blue magnitudes taken from the literature, and thus they have not been measured in the same manner as the apparent magnitudes calculated here. Since the galaxies selected with regard to far-IR color, but without an optical bias, still give correlations of $L_{\text {FIR }}$ with optical luminosity that are consistent with what we find for all 30 ICSS FIRGs, we conclude that optical selection effects are not responsible for the correlation seen in Figure $9 d$. In fact, all the data subsets mentioned above have far-infrared luminosities that increase approximately as the 1.5 power of the optical luminosity. We note that an optically selected sample of " normal" galaxies would fall below the best fit line in Figure $9 d$. The ICSS FIRGs therefore form an upper bound to more quiescent galaxies in the $\log L_{\mathrm{FIR}}, M_{R}$ plane.

In Figure 10 we see the same emission-line parameters as in Fig. 9, but here they are plotted against the nuclear $R$ band magnitudes from Table 1 . There is a very good correlation of the nuclear $R$ magnitude with the nuclear $\mathrm{H} \alpha+[\mathrm{N}$ II $]$ luminosity (slope $=-0.60$, implying $L_{\mathrm{NUC}} \propto L_{R}^{1.50 \pm 0.37}$ ).

Given the good correlations seen in Figures 9 and 10, we might expect to see a relation between $r_{e}$, the emission-line half-light radius, and both the $R$ band absolute magnitude and the far-infrared luminosity. In Figure 11 we plot $\log r_{e}$ against $\log L_{\text {TOT }}, \log L_{\mathrm{FIR}}, M_{R}$, and total $\log \mathrm{EQW}_{\mathrm{H} \alpha+[\mathrm{N} \mathrm{II}]}$, respectively. Lines indicating relatively high and low $\mathrm{H} \alpha+[\mathrm{N}$ II $]$ surface brightness are also indicated in Figure 11a. The ICSS FIRG Mrk 231, known to have a type 1 Seyfert nucleus, is labeled in Figures $11 a-11 c$. It is interesting that $r_{e}$ correlates neither with $M_{R}$ nor with $L_{\text {TOT }}$. While there is no correlation of $r_{e}$ with the total emission-line or continuum luminosity, there is a rather good correlation of $r_{e}$ with $L_{\mathrm{FIR}}$. Neglecting for the moment the point representing Mrk 231, we have $L_{\text {FIR }} \propto$ $r_{e}^{1.7 \pm 0.22}$ (shown by a dashed line in Fig. $11 b$ ). This is the same power-law slope, within the errors, found for the correlations of $L_{\mathrm{FIR}}$ with $L_{R}$ and with $L_{B}$ (see above). Notice that the $\mathrm{H} \alpha+[\mathrm{N}$ II $]$ half-light radius correlates better with $L_{\mathrm{FIR}}$ than 

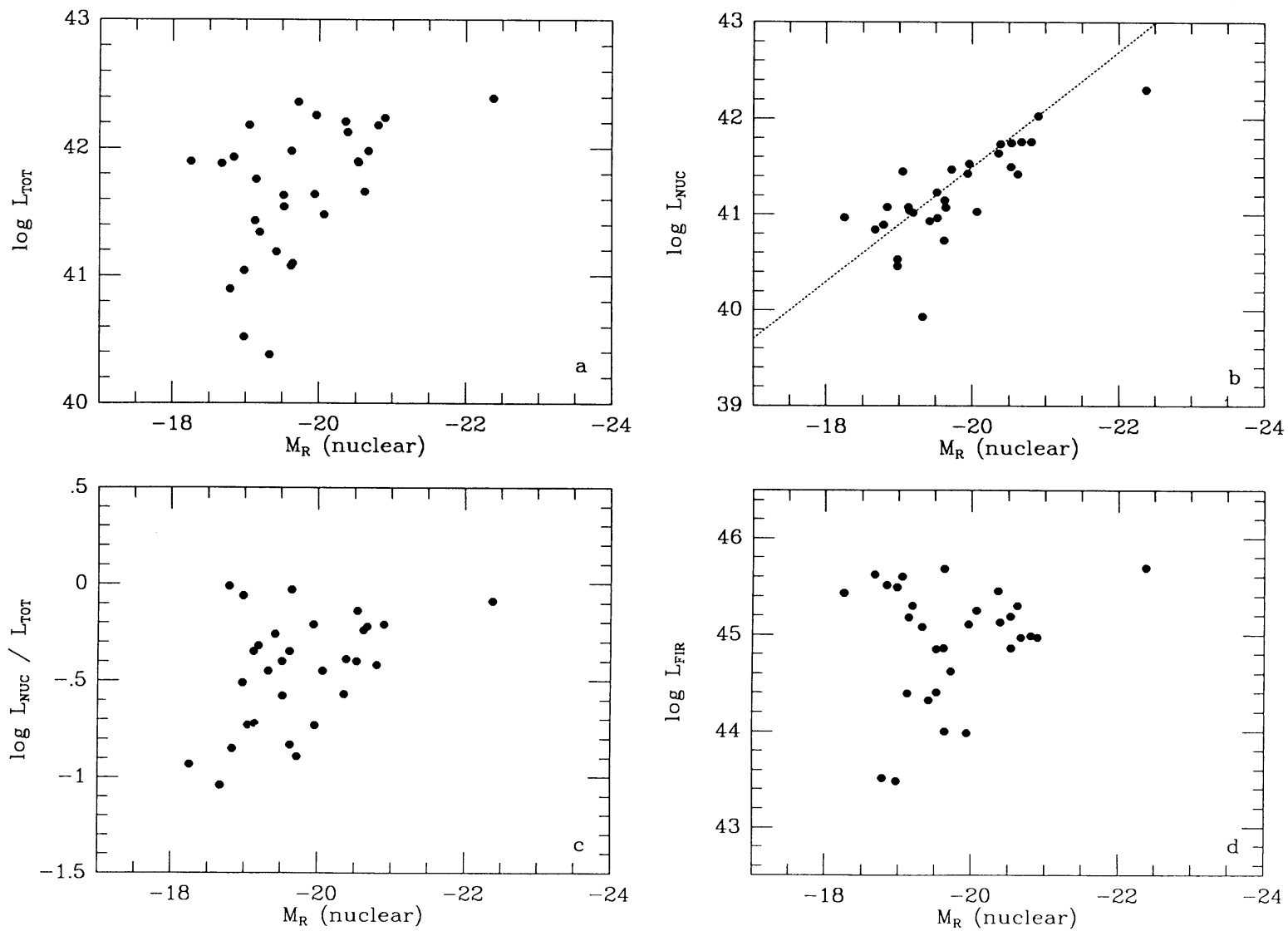

FIG. 10. - The same as in Fig. 9, except the nuclear absolute $R$ band magnitude is now plotted on the abcissa
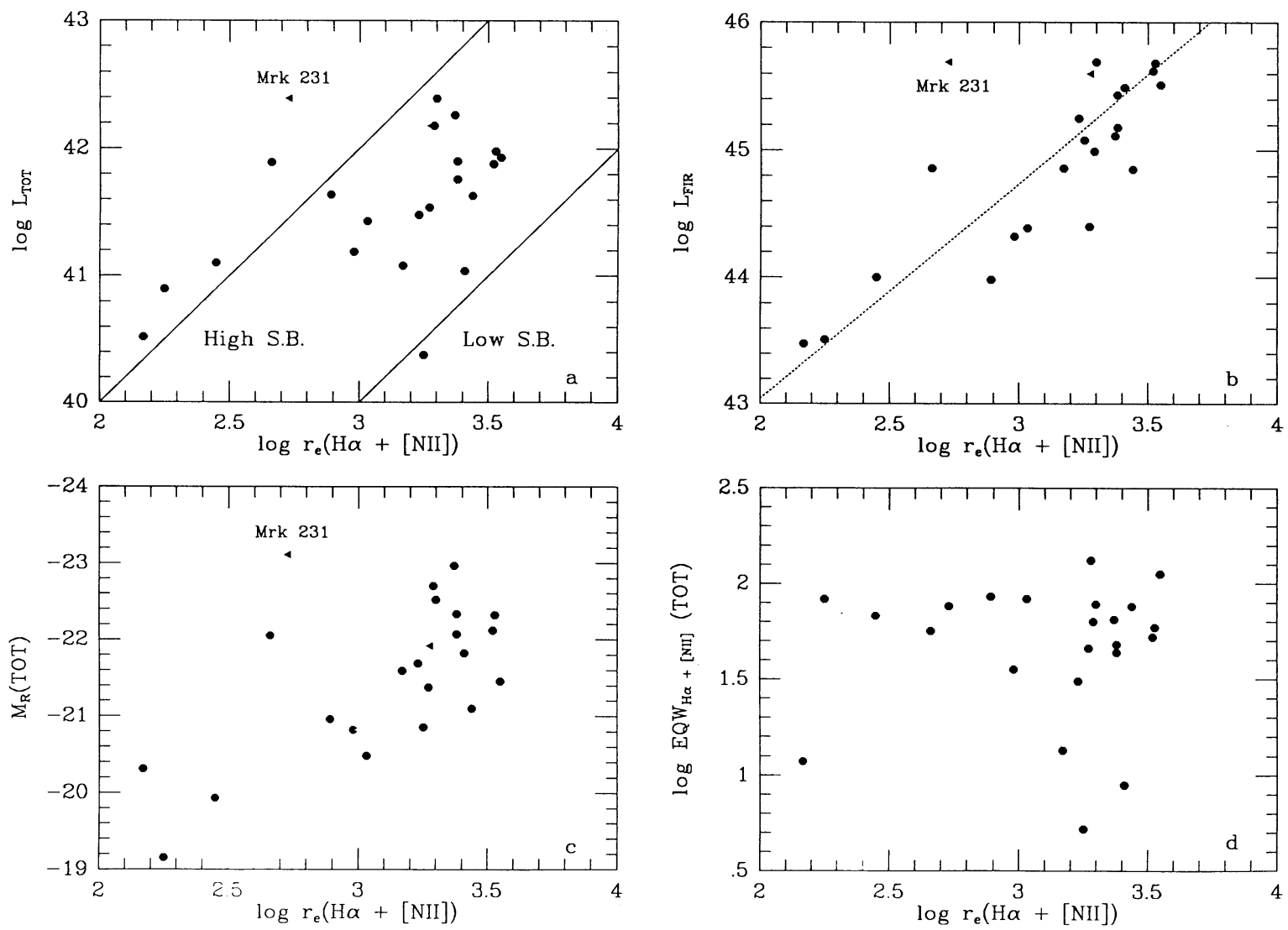

FIG. 11.-Plot of the $\mathrm{H} \alpha+[\mathrm{N}$ II $]$ half-light radius, $r_{e}(\mathrm{pc})$ against $(a)$ the total $\mathrm{H} \alpha+[\mathrm{N}$ II $]$ luminosity, $(b)$ the far-infrared luminosity, $(c)$ the absolute $R$ band

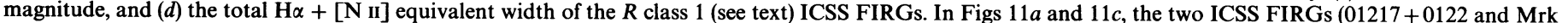

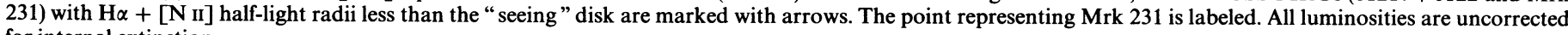
for internal extinction. 
does $L_{\text {TOT }}$. This may be because the $\mathrm{H} \alpha+[\mathrm{N}$ II $]$ luminosity is affected more by dust than is the half-light radius.

If we subtract the nuclear $\mathrm{H} \alpha+[\mathrm{N}$ II $]$ and continuum fluxes from the total $\mathrm{H} \alpha+[\mathrm{N}$ II $]$ and continuum fluxes we arrive at the extranuclear $(r \geq 1 \mathrm{kpc})$ fluxes. A plot of $L_{\text {extr }}(\mathrm{H} \alpha+[\mathrm{N} \mathrm{II}])$ versus $M_{R}$ (extr) is shown in Figure 12a. A linear least-squares fit to these points yields $L_{\text {extr }}\left(\mathrm{H} \alpha+\left[\mathrm{N}_{\text {III }}\right]\right) \propto L_{R}^{1.62 \pm 0.22}$. In Figure $12 b$, we plot the $\log$ of the nuclear and extranuclear $\mathrm{H} \alpha+[\mathrm{N}$ II $]$ luminosities versus the nuclear and extranuclear $R$ band magnitudes, respectively, for all the ICSS FIRGs. While the lines are nearly parallel, the extranuclear $R$ magnitudes are displaced from the nuclear $R$ magnitudes by $\sim 1.5 \mathrm{mag}$ (at a

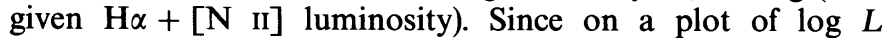
$(\mathrm{H} \alpha+[\mathrm{N}$ II $])$ versus $\log L(R)$, lines of unit slope are equivalent to lines of constant $\mathrm{H} \alpha+[\mathrm{N}$ iI $]$ equivalent width, the displacement of the extranuclear points to larger $M_{R}$ implies the extranuclear gas has a smaller $\mathrm{H} \alpha+[\mathrm{N}$ II $]$ equivalent width. In fact, the ICSS FIRGs have a mean nuclear log EQW $\mathrm{EW}_{\mathrm{H} \alpha[\mathrm{N} \mathrm{II]}}(\AA)=$ $2.02(\sigma=0.32)$ and a mean extranuclear log $\operatorname{EQW}_{\mathrm{H} \alpha+[\mathrm{N} \mathrm{II}}(\AA)=1.48(\sigma=0.42)$. The emission-line gas is
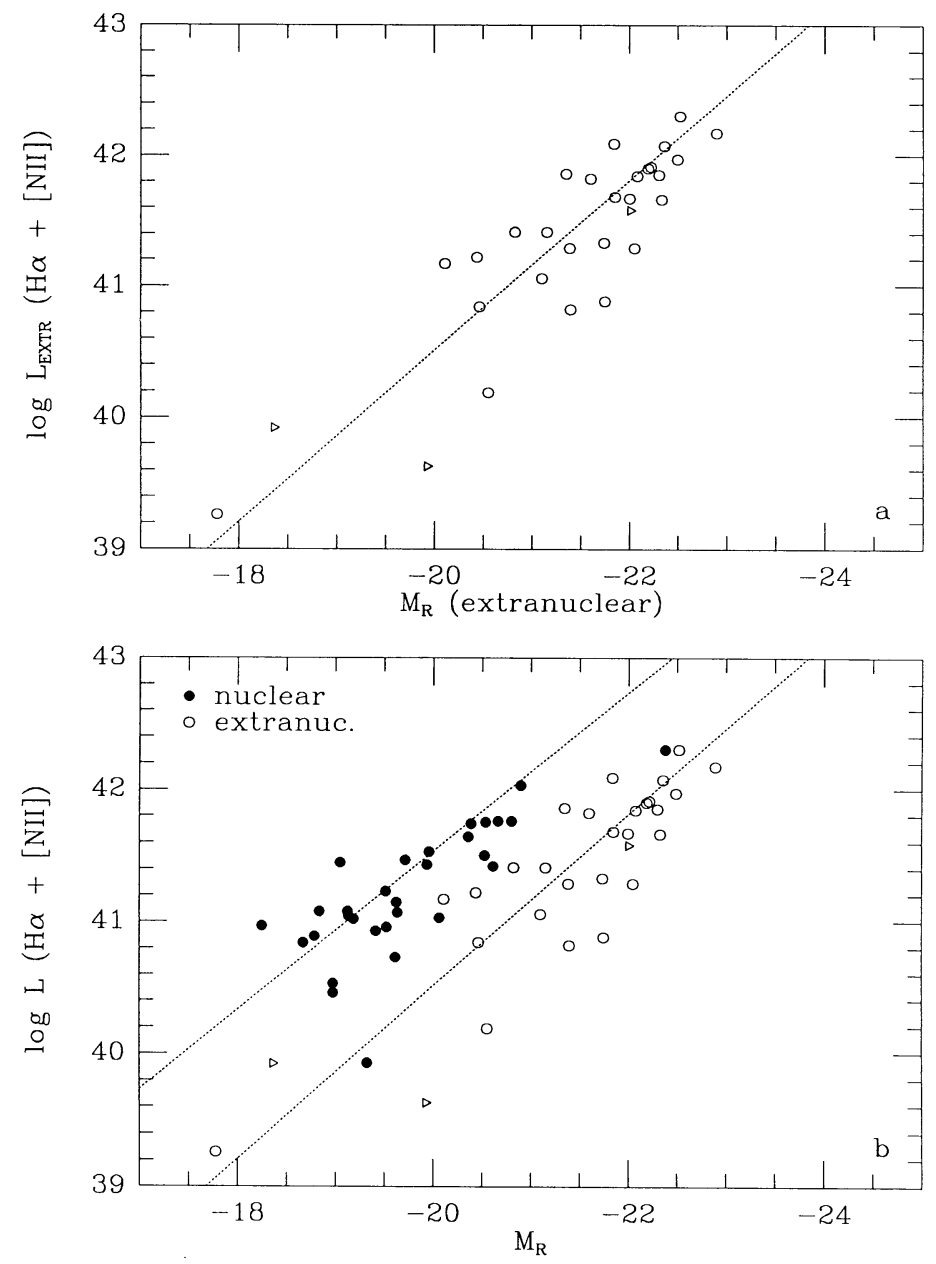

FIG. 12.- (a) Plot of the extranuclear (defined in the text) absolute $R$ band magnitude against the extranuclear $\mathrm{H} \alpha+[\mathrm{N}$ II] luminosity for the ICSS FIRGs. The linear least-squares fit to the data is drawn as atted line. (b) Plot of the nuclear (solid) and extranuclear (open) absolute $R$ band magnitudes and $\mathrm{H} \alpha+\left[\mathrm{N}_{\mathrm{II}}\right]$ luminosities for the ICSS FIRGs. As above, the linear leastsquares fits to the data are drawn as dotted lines. The arrows indicate lower limits to the $R$ band absolute magnitudes. The $\mathrm{H} \alpha+[\mathrm{N}$ II] and $R$ band luminosities are uncorrected for internal extinction. more centrally concentrated than the starlight in our sample galaxies. This could be explained either by a higher covering factor in the nucleus (allowing more efficient conversion of Lyman-continuum photons to $\mathrm{H} \alpha$ photons), or by the presence of enhanced nuclear star formation. The majority of the ICSS FIRGs have more than $50 \%$ of their flux originating beyond 1 $\mathrm{kpc}$ from the nucleus in both $R$ and $\mathrm{H} \alpha+[\mathrm{N}$ II] .

The ICSS FIRGs have a mean total $\log \mathrm{EQW}_{\mathrm{H} \alpha+[\mathrm{N} \mathrm{II]}}(\AA)=$ $1.69(\sigma=0.34)$. The Sc galaxies of KK (1983) typically have $\log \mathrm{EQW}_{\mathrm{H} \alpha+[\mathrm{N} \mathrm{II}]}(\AA)=1.4-1.5$, with the most luminous in $\mathrm{H} \alpha+[\mathrm{N}$ II $]$ having $\log \mathrm{EQW}_{\mathrm{H} \alpha+[\mathrm{N} \mathrm{III}}(\AA)=1.7$. Our sample galaxies then, have $\mathrm{H} \alpha+[\mathrm{N}$ II $]$ equivalent widths comparable to the brightest Sc galaxies. Kennicutt (1983) uses the $\mathrm{H} \alpha$ equivalent width and the broad-band colors to constrain the IMF and hence estimate the current star formation rate in a sample of spiral and irregular galaxies. Using an extended Miller-Scalo IMF (Miller and Scalo 1979), where $\Psi(m) \propto m^{-1.4} \quad\left(0.1 \leq m \leq 1 M_{\odot}\right) \quad$ and $\quad \Psi(m) \propto m^{-2.5}$ $\left(1 \leq m \leq 100 M_{\odot}\right)$, he finds that the present star formation rate (SFR) in late-type galaxies is comparable to the average past SFR in the disks (determined from the stellar mass and the age of the disks). The largest SFR for the Sc galaxies is $\sim 10 M_{\odot}$ $\mathrm{yr}^{-1}$ (for $H_{0}=75 \mathrm{~km} \mathrm{~s}^{-1} \mathrm{Mpc}^{-1}$ ). A continuous SFR could then also explain the mean total $\mathrm{H} \alpha+[\mathrm{N}$ II $]$ equivalent width that we have measured in our ICSS FIRGs without having to invoke a burst of star formation.

Both the differential reddening of the stars and gas and the underlying stellar absorption might affect our measured $\mathrm{H} \alpha+[\mathrm{N}$ II $]$ equivalent widths. Since we have not yet fully analyzed the long-slit spectral data, we can estimate the error in $\mathrm{EQW}_{\mathrm{H} \alpha+[\mathrm{N} \mathrm{II]}}$ only in the nuclei of our FIRGs. Here, through the linear correlation of continuum color with the Balmer decrement, we know that the stars and the gas are reddened by the same material (see Armus, Heckman, and Miley 1989). Also, the Balmer absorption-line equivalent widths in the nuclei of the FIRGs with the strongest absorption are $<10 \AA$, implying that these stellar features are causing us to underestimate the emission-line equivalent width at $\mathrm{H} \alpha$ by at most $10 \%$. The extranuclear, and hence the total, equivalent widths of our ICSS FIRGs may be affected by either reddening or stellar absorption lines, and therefore the apparent similarity of mean total $\mathrm{EQW}_{\mathrm{H} \alpha+[\mathrm{N} \text { II] }}$ to that found for giant SC galaxies should be viewed cautiously.

Taken at face value, then, the total $\mathrm{EQW}_{\mathrm{H} \alpha+[\mathrm{N} \mathrm{II}]}$ of our ICSS FIRGs does not rule out continuous star formation. The star formation rate derived from the far-infrared luminosity, however, is much higher than that derived from the $\mathrm{H} \alpha$ luminosity. From Hunter and Gallagher (1986) and Hunter et al. (1986), we have $\mathrm{SFR}=7.1 \times 10^{-42} L_{\mathrm{H} \alpha}$, and $\mathrm{SFR}=1.3$ $\times 10^{-43} L_{\text {FIR }} M_{\odot} \mathrm{yr}^{-1}$, for a Salpeter IMF between 0.1 and $100 M_{\odot}$. Correcting our mean $L_{\text {TOт }}(\mathrm{H} \alpha+[\mathrm{N} \mathrm{II}])$ for the median $\log [\mathrm{N} \mathrm{II}] / \mathrm{H} \alpha$ (nuclear) ratio $=-0.23$, gives a mean $\mathrm{SFR} \sim 2 \mathrm{M}_{\odot} \mathrm{yr}^{-1}$ uncorrected for internal reddening. From the mean $L_{\text {FIR }}$ of our ICSS FIRGs we calculate a mean SFR $\sim 117 M_{\odot} \mathrm{yr}^{-1}$. Thus the far-infrared derived SFR is a factor of $\sim 60$ greater than the $H \alpha$-derived SFR. The typical extinction at $\mathrm{H} \alpha$ as derived from the $\mathrm{H} \alpha / \mathrm{H} \beta$ ratio measured in our nuclear spectra is $\sim 2 \mathrm{mag}$ (see Armus, Heckman, and Miley 1989). If we correct our $\mathrm{H} \alpha$ luminosities for $2.0 \mathrm{mag}$ of extinction, the far-infrared-derived SFR is still nearly an order of magnitude larger than the $\mathrm{H} \alpha$-derived SFR.

Since the Balmer decrement measures the reddening to the least heavily obscured material, it is quite possible that we have 
substantially underestimated the corrected $\mathrm{H} \alpha$ luminosity, and hence the SFR. To get a better estimate of the "true" extinction, we have measured the $\mathrm{H} \alpha$ fluxes within a 5 ".5 diameter aperture for the seven galaxies which have $\mathrm{Br} \alpha$ fluxes as given in DePoy (1987). Comparing the $\mathrm{H} \alpha / \mathrm{Br} \alpha$ ratio to that expected from case B recombination at $T=10^{4} \mathrm{~K}$ and $n=10^{2} \mathrm{~cm}^{-3}$ I ( 35.7) we can derive an extinction in magnitudes at $\mathrm{H} \alpha$. This extinction $A_{\mathrm{H} \alpha}$ is on average a full magnitude greater than that derived from the $\mathrm{H} \alpha / \mathrm{H} \beta$ ratio, indicating that the $\mathrm{H} \alpha$ luminosity of our ICSS FIRG, and therefore the SFR derived from this luminosity, may be a factor of $\sim 3$ greater than quoted above. We arrive at similar factors when comparing the $\mathrm{Br} \alpha$ flux of M82 (Rieke et al. 1980) to the $\mathrm{H} \alpha$ flux we measure in three apertures of $8^{\prime \prime}, 11^{\prime \prime}$, and $30^{\prime \prime}$ diameter. Using the Br $\alpha$ data as a guide and applying a correction for $\sim 3 \mathrm{mag}$ of extinction at $\mathrm{H} \alpha$, the SFR as derived from the far-infrared luminosity is only a factor of $\sim 3$ greater than that derived from the $\mathrm{H} \alpha$ luminosity (on average) for the ICSS FIRGs. Given the modeldependent uncertainty in the two formulae from Hunter and Gallagher (1986) and Hunter et al. (1986), this factor of $\sim 3$ is probably not significant.

\section{b) Galactic Environment}

The complex, large-scale emission-line nebulae of many of our sample galaxies, along with the well-known link between galactic interactions and enhanced $\mathrm{H} \alpha$ emission (Keel et al. 1985; Kennicutt et al. 1987; BLW), has led us to attempt a classification of these galaxies with respect to their $R$ band continuum images. We make these entirely subjective classifications in an effort to shed some light on the environmental circumstances that may have resulted in the emission-line nebulosities seen in Figure 1.

The broad-band morphologies of our IR color-selected galaxies fall into three groups. The first group ( $R$ class 1$)$ consists of galaxies with one stellar system and one nucleus. This is by far the most populous category, containing 23 IR colorselected galaxies, along with NGC 660, NGC 2146, NGC 3079, NGC 4102, NGC 4536, and NGC 5900. A great number of these ICSS $R$ class 1 galaxies $(\sim 75 \%)$ display morphological peculiarities that may be evidence of some past interaction/ merger, but the main bodies of the participants seem to have coalesced into a single system. Deeper broad- and narrowband imaging of the remaining $25 \%$ of the IR color-selected $R$ class 1 galaxies may reveal faint tails or loops indicative of some past interaction. If these relatively "normal" looking galaxies have never undergone such an interaction, then another triggering mechanism must be found for the starburst and/or AGN that powers their warm IR colors and bright extended $\mathrm{H} \alpha$ emission.

The second major group ( $R$ class 2 ) consists of galaxies that appear to have only one large-scale stellar system, but multiple nuclei. The IR color-selected galaxies that fit this description are NGC 3690, 08572 + 3915, $15163+4255$, and $17132+5313$. The galaxy Mrk 266, which does not meet our IR color criteria, belongs in this group as well. This category is the hardest to define, in the sense that the galaxies must contain secondary peaks/inner structure made up of more than one galactic nucleus, but not simply a dominant nucleus with associated giant $\mathrm{H}$ II regions or hot spots. The fact that this group contains the fewest members partially reflects our caution in assigning galaxies to this class but also may indicate the speed at which the nuclei of galaxies merge once they lie close together within a common envelope.
Finally, we have objects such as $13136+6223$ that are composed of two distinct galactic systems apparently involved in an interaction. Evidence for this interpretation comes from the presence of distorted spiral disks, long (tens of $\mathrm{kpc}$ ) opposing tidal tails, and bridges of luminous matter connecting the nuclei of the individual systems. These galaxies will hereafter be designated as $R$ class 3 , and other galaxies in this group are $10565+2448$ and $12018-1814$. We have spectra of both of the galaxies in two of these systems $(13136+6223$ and $10565+2448)$, indicating that they are indeed interacting, with velocity differences of 180 and $115 \mathrm{~km} \mathrm{~s}^{-1}$, respectively. Two galaxies not chosen for their IR colors, NGC 1667 and NGC 2798 , can be placed in this category as well.

Before we can compare the $R$ class 1 galaxies to those of the $R$ class 2 and $R$ class 3 in an attempt to discover a link between local environment and emission-line properties, we must first determine if the $R$ class 1 galaxies are indeed isolated. To do this we have searched the individual CCD frames for nonstellar objects of comparable brightness. As mentioned in $\S$ II, the field of view for all the galaxies was $\sim 2.5 \times 2.5$ (except in the case of Arp 220 and NGC 6240 where it was $4^{\prime} \times 4^{\prime}$ ). Some galaxies were not placed at the center of the field of view, however, in order to avoid saturation by nearby bright stars. For these galaxies, and for nearby galaxies that filled the field of view, we went back to the Palomar Sky Survey and the ESO Southern Sky Survey to find the nearest galactic object. Most of our $R$ class 1 galaxies have no other galaxy of comparable brightness within a projected distance of $50 \mathrm{kpc}$. Many galaxies have no neighbors for at least $100 \mathrm{kpc}$ (in projected distance). There are three galaxies $(01217+0122,05447-2114$, and $15250+3609$ ) with neighboring galaxies less than $30 \mathrm{kpc}$ away in projection. For $05447-2114$, there is no sign of an interaction in $R$ or $\mathrm{H} \alpha+[\mathrm{N}$ II $]$. For $0217+0122$ the companion galaxy is not at the same redshift (see above). For $15250+3609$ the companion galaxy has no detectable $\mathrm{H} \alpha+[\mathrm{N}$ II $]$ emission down to $10^{-16}$ ergs $\mathrm{cm}^{-2} \mathrm{~s}^{-1} \operatorname{arcsec}^{-2}$ and may also be at a different redshift than the primary galaxy. In summary, then, our $R$ class 1 galaxies display no evidence in their optical broad-band images for being currently involved in galactic interactions, despite often being morphologically peculiar. ${ }^{1}$

The $R$ class 1 galaxies are plotted in the $L_{\text {FIR }}, L_{\text {TOT }}(H \alpha$ $+[\mathrm{N} \mathrm{II}])$ plane in Figure 13. As in Figure 4, the locus of isolated spiral and irregular galaxies taken from KK (1983) is given by a dashed curve. The $R$ class 1 ICSS FIRGs span the range in $L_{\text {FIR }}, L_{\text {TOT }}$ that we see for all of the IR color-selected galaxies. The mean values of $\log L_{\text {FIR }}$ and $\log L_{\text {TOT }}$ are $=44.90$ $(\sigma=0.68)$ and $=41.59(\sigma=0.56)$, respectively, for the $R$ class 1 galaxies. The average total emission-line $\mathrm{H} \alpha+[\mathrm{N}$ II $]$ luminosity, then, is about 4 times that found for the isolated galaxies of Kennicutt and Kent (1983). This difference is significant at greater than the $99 \%$ confidence level.

As mentioned above, most $(75 \%)$ of the ICSS FIRGs that belong to $R$ class 1 seem also to have quite distorted broadband morphologies. The $\mathrm{H} \alpha+[\mathrm{N}$ II $], R$-band, and far-infrared luminosities of these galaxies are not significantly different

\footnotetext{
${ }^{1}$ We have classified the prototype FIRG M82 as an $R$ class 1 galaxy. Although the optical continuum image of M82 shows no obvious signs of an ongoing galactic interaction, there is a clumpy $\mathrm{H}$ I bridge connecting M82 to its nearest neighbor, M81 (Cottrell 1977; Gottesman and Weliachew 1977). The envelope of atomic hydrogen encompassing the M82, M81 system is interpreted as the result of a hyperbolic encounter approximately $2 \times 10^{8} \mathrm{yr}$ ago, having a perigalactic distance of $\sim 9 \mathrm{kpc}$ (Cottrell 1977). Much of the $\mathrm{H}$ gas now surrounding M82 may have been captured during that encounter.
} 


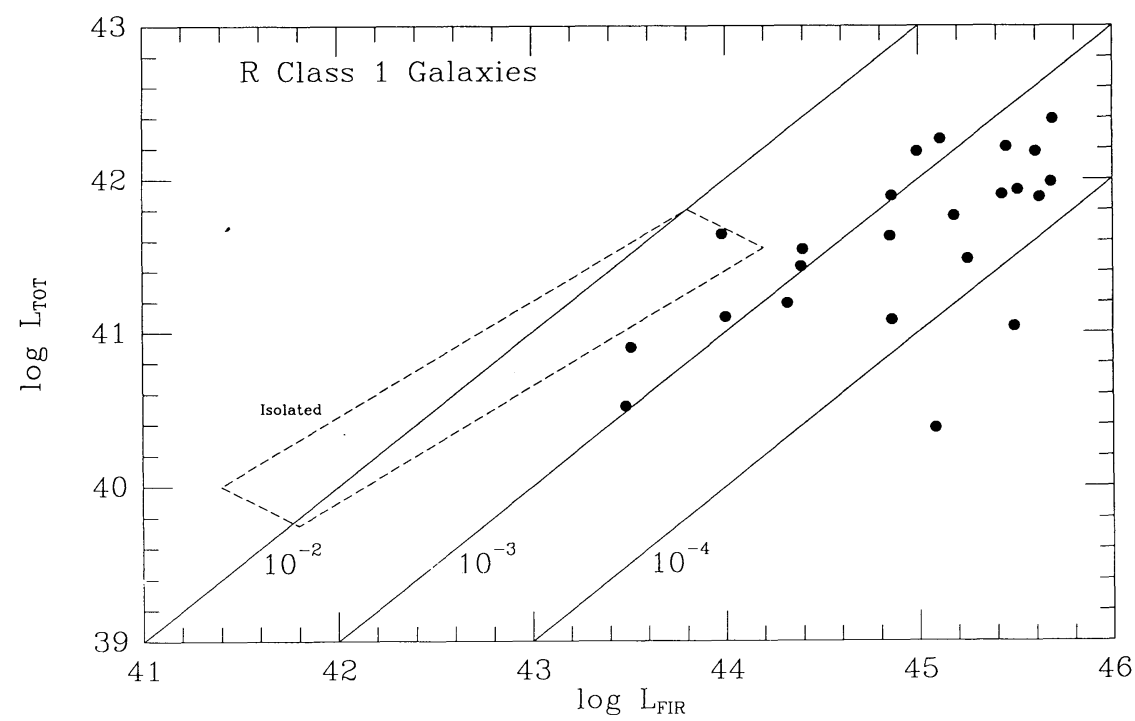

Fig. 13.-Same as in Fig. 4, except for the $R$ class 1 (defined in text) ICSS FIRGs only. Also, the loci of isolated spiral galaxies taken from Kennicutt and Kent (1983) is shown as a dotted line.

from the small subset of undisturbed $R$ class 1 galaxies. The mean redshifts of the two groups are also not significantly different, implying that it is not the greater distance of the latter group that is preventing us from viewing their distorted morphologies. It is possible that the undisturbed $R$ class 1 galaxies have not suffered quite as damaging interactions, or that the obvious signs of past encounters have already disappeared. Deeper images of these galaxies may uncover the faint signs of such an interaction.

In systems with more than one nucleus, the $\mathrm{H} \alpha+\left[\begin{array}{ll}\mathrm{N} & \mathrm{II}\end{array}\right]$ emission is not distributed evenly between the nuclei. One nucleus always dominates the other in $\mathrm{H} \alpha+[\mathrm{N}$ II $]$, with a mean $\log L_{\mathrm{NUC} 1} / L_{\mathrm{NUC} 2}=1.10(\sigma=0.13)$ for the $R$ class 3 galaxies and a mean $\log L_{\mathrm{NUC} 1} / L_{\mathrm{NUC} 2}=0.62(\sigma=0.25)$ for the $R$ class 2 galaxies. ${ }^{2}$ We see no linear trend between projected nuclear separation and relative emission-line flux. Compared to the $R$ class 3 galaxies, the $R$ class 2 galaxies have their $\mathrm{H} \alpha+\left[\begin{array}{ll}\mathrm{N} & \mathrm{II}\end{array}\right]$ emission more evenly divided between their nuclei, but small number statistics are probably dominating here. This may also be a selection effect in the sense that merged galaxies with larger value of $L_{\mathrm{NUC1}} / L_{\mathrm{NUC2}}$ would be classified as $R$ class 1 , with the second $\mathrm{H} \alpha+[\mathrm{N}$ II $]$ peak being called an extranuclear $\mathrm{H}$ il region or hot spot. For the $R$ class 3 and $R$ class 2 galaxies, the nuclei that are more luminous in $\mathrm{H} \alpha+[\mathrm{N} \mathrm{II}]$ are also more luminous in the $R$ band. The mean differnce in $M_{R}$ for the nuclei in the $R$ class 3 systems is $0.7 \mathrm{mag}$ (a factor of 1.9), while the mean difference in $M_{R}$ for the nuclei in the $R$ class 2 systems is 0.6 mag (a factor of 1.7 ). It is likely that the ratio of emission-line flux between nuclei in an interaction/merger is a complicated function of orbital parameters and individual galactic properties such as total mass and gas content.

An intriguing explanation for the diverse properties that we see in the ICSS FIRGs is that we are looking at these galaxies

\footnotetext{
${ }^{2}$ Due to the ambiguity concerning the position of the true nuclei in the NGC 3690 system, we have taken the sum of the $\mathrm{H} \alpha+[\mathrm{N} \mathrm{II}]$ luminosity of nucleus a (labeled as C by Gehrz, Sramek, and Weedman 1983), and nucleus c (labeled as B by Gehrz, Sramek, and Weedman 1983) as $L_{\text {NUC1 }}$. The $\mathrm{H} \alpha+[\mathrm{N} \mathrm{II}]$ luminosity of our nucleus b (labeled as A by Gehrz, Sramek, and Weedman 1983) we refer to as $L_{\mathrm{NUC2}}$.
}

at different stages in their evolution. The simplest picture would be one in which the $R$ class 3 galaxies evolved into the $R$ class 2 galaxies, which then evolved into the $R$ class 1 galaxies.

Morphologically, this is easy to envision, as the $R$ class 3 galaxies appear to be in the early stages of often quite damaging interactions, whereas the $R$ class 1 galaxies have apparently completed the merging process. The $R$ class 2 galaxies would then fit nicely into the intermediate stages of this scenario. Without detailed simulations which take into account, for example, the effects of massive galactic halos, we cannot be certain that the $R$ class 3 galaxies will indeed merge. It seems unlikely though, that galaxies undergoing such severe interactions can escape such a fate.

Energetically, we would expect the currently interacting/ merging galaxies to be brighter than the older, fully merged galaxies, if the interaction process itself is responsible for the enhanced emission. While we can group the $R$ class 2 and $R$ class 3 galaxies (currently interacting) together for a comparison to the $R$ class 1 galaxies, the small number of the former galaxies will greatly influence the results. Nonetheless, if we make such a comparison we find that the mean far-infrared, $\mathrm{H} \alpha+[\mathrm{N} \mathrm{II}]$, and $R$ band luminosities of the currently interacting galaxies are all a factor of $\sim 2-2.5$ greater than those measured for the $R$ class 1 galaxies. Due to the small number of currently interacting galaxies, the differences in the means are not statistically significant. We find the same result if we restrict our comparison to the brightest member (in $\mathrm{H} \alpha+[\mathrm{N} \mathrm{II}]$ and $R$ ) of each interacting pair, indicating that the $R$ class 2 and $R$ class 3 systems are not brighter in total luminosity solely because they are composed of two galaxies.

An alternative explanation is that all powerful far-infrared galaxies may go through a period during which they have warm globally averaged dust temperatures. If the FIRG phenomenon is directly related to galaxy interactions/mergers (which it seems to be from our data, as well as those of Sanders et al. 1988), then the time over which the average dust temperature is elevated may depend critically upon the parameters of the encounter. In this scenario the $R$ class 3 ICSS FIRGs would not evolve into the $R$ class 1 ICSS FIRGs. The different broad-band morphologies would than represent a range of 
galactic encounters, each being capable of raising the average dust temperature of the galaxy (galaxies) involved. It is interesting, then, that we see so few ICSS FIRGs that are currently interacting with a conspicuous companion galaxy. In selecting galaxies with warm dust temperatures, we may be preferentially selecting galaxies that have experienced quite inelastic encounters and have recently merged. This might explain the lack of companions in $75 \%$ of the galaxies. Only interacting galaxies whose orbits cause them to merge quickly develop far-infrared flux ratios that meet our selection criteria. The ICSS FIRGs may represent the precursors of more classical forms of activity such as QSOs (see Sanders et al. 1988). Imaging of the remainder of the ICSS FIRG sample will help bolster our statistics and enable a stricter evaluation of this hypothesis.

\section{CONCLUSIONS}

In an attempt to determine the luminosities, morphologies, and ultimate energy source of the emission-line nebulae that reside within powerful far-infrared galaxies (FIRGs), we have analyzed the narrow-band $\mathrm{H} \alpha+[\mathrm{N}$ II] images of 30 galaxies selected to have far-infrared spectral energy distributions similar to the prototype FIRGs Arp 220, NGC 6240, and M82. The contour plots (Fig. 1) and gray-scale images (Fig. 8) of these IR color-selected sample (ICSS) galaxies have morphologies that range from smooth and structureless to highly complex and chaotic, with some showing evidence for ordered structure on scales of a few $\mathrm{kpc}$ to tens of kpc. Of the seven ICSS FIRGs that are apparently highly flattened in $R$ (majorto-minor axis ratio $\geq 2.0$ ), six have $\mathrm{H} \alpha+[\mathrm{N}$ II] isophotes that are either more circular than, or perpendicular to, their continuum isophotes. This structure may represent emission-line gas that is extended out of the plane of the galaxy. This also seems to be the case in three non-ICSS disk galaxies viewed nearly edge-on. Overall, the ICSS FIRG emission-line nebulae are quite large, having a mean diameter (maximum extent) of $\sim 16$ $\mathrm{kpc}$ and an average half-light radius, $r_{e} \sim 1.3 \mathrm{kpc}$ (the latter value only applies to the ICSS FIRGs that are single-galaxy systems). Typically $\frac{2}{3}$ of the total $\mathrm{H} \alpha+[\mathrm{N}$ II $]$ emission originates from beyond a radius of $1 \mathrm{kpc}$. A comparison of the nuclear and extranuclear $\mathrm{H} \alpha+[\mathrm{N}$ II] equivalent widths (105 vs. $30 \AA$, respectively) implies that the emission-line gas is more centrally concentrated than the starlight in our sample galaxies.

The mean total $\mathrm{H} \alpha+[\mathrm{N}$ II] luminosity of the FIRGs ( $\sim 5 \times 10^{41} \mathrm{ergs} \mathrm{s}^{-1}$, uncorrected for internal extinction) is a factor of 2-3 greater than that of individual members of pairs of optically selected interacting spirals (BLW 1988) and a factor of $\sim 5$ greater than the $\mathrm{H} \alpha+[\mathrm{N}$ II] luminosity of isolated spiral galaxies in general (KK 1983). The typical uncorrected $\mathrm{H} \alpha+\left[\begin{array}{ll}\mathrm{N} & \mathrm{II}\end{array}\right]$ luminosities of the ICSS FIRGs are comparable to those of the most luminous known (noninteracting) spiral galaxies. The average internal extinction is $\sim 3 \mathrm{mag}$ at $\mathrm{H} \alpha$ in the ICSS FIRGs (using available $\mathrm{Br} \alpha / \mathrm{H} \alpha$ flux ratios). Thus, the log-average intrinsic (extinction-

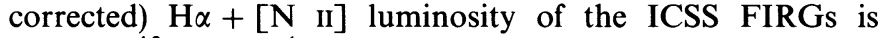
$\sim 8 \times 10^{42}$ ergs $\mathrm{s}^{-1}$. Since the typical extinction in normal giant $\mathrm{H}$ II regions in spiral galaxies is only $\sim 1 \mathrm{mag}$ at $\mathrm{H} \alpha$ (Kennicutt 1983), typical ICSS FIRGs have intrinsic $\mathrm{H} \alpha+[\mathrm{N} \mathrm{II}]$ luminosities that are a factor of $\sim 30$ greater than average noninteracting spiral galaxies and a factor of $\sim 6$ greater than those of the most luminous known noninteracting spiral galaxies.

Only $\sim 25 \%$ of the nearby $(z \leq 0.10)$ FIRGs have morphologies suggesting that large $\mathrm{H}$ II regions contribute significantly to their emission-line appearance. While previous studies of the ICSS FIRGs suggest that young stars are dominating their optical spectra, a majority of their emission-line nebulae are evidently not powered by hot, young stars distributed in the same manner as they are in normal spiral galaxies (within individual giant $\mathrm{OB}$ associations).

If young stars are indeed responsible for the far-infrared and $\mathrm{H} \alpha+[\mathrm{N}$ II $]$ emission, then we can compare the star formation rates (SFRs) implied by these luminosities. After correcting for [N II] emission in the bandpass and a typical extinction of 2.0 mag at $\mathrm{H} \alpha$, as implied by our measured $\mathrm{H} \alpha / \mathrm{H} \beta$ ratios, the total SFR calculated from the far-infrared luminosity is nearly an order of magnitude greater than that derived from the $\mathrm{H} \alpha$ luminosity. Comparing the extinction at $\mathrm{H} \alpha$ derived from the $\mathrm{H} \alpha$-to-H $\beta$ flux ratio to that derived from the $\mathrm{H} \alpha$-to-Br $\alpha$ flux ratio for the eight galaxies that we have in common with DePoy (1987) and Rieke et al. (1980), we find that the $\mathrm{H} \alpha$-to$\mathrm{H} \beta$ ratio is underestimating the extinction at $\mathrm{H} \alpha$ by typically $\sim 1$ mag. If we use this as an indication of the amount that we have underestimated the extinction at $\mathrm{H} \alpha$ for the ICSS FIRGs as a class, the SFR derived from the far-infrared luminosity is still greater (but only by a factor of $\sim 3$ ) than the SFR derived from the $\mathrm{Br} \alpha$-corrected $\mathrm{H} \alpha$ luminosity. This may be indicating a steeper IMF or a smaller upper mass cutoff than used in the models of Hunter and Gallagher (1986) and Hunter et al. (1986). Alternatively, since the $\mathrm{H} \alpha$ and the far-infrared luminosity effectively measure the SFR over different periods of time (the $\mathrm{H} \alpha$ measuring the current SFR, and the far-IR essentially measuring the SFR over the past $\sim 10^{8}-10^{9} \mathrm{yr}$ ), a declining SFR for the ICSS FIRGs may be indicated. At this point, it is not possible to rule out any of these explanations from our available data.

The fact that we see few ICSS FIRGs $(10 \%)$ that are currently interacting with a conspicuous companion galaxy yet many $(80 \%)$ that are highly distorted in continuum morphology suggests that our IR color criteria are preferentially selecting galaxies that have recently undergone a merger.

The good correlation of both $\mathrm{H} \alpha+[\mathrm{N}$ II] luminosity and far-infrared luminosity with absolute $R$ magnitude along with spectroscopic evidence that the optical continuum is predominantly produced by young stars (Armus, Heckman, and Miley 1987, 1989), implies that the emission-line and far-infrared luminosity are both powered by young stars. Because the morphology of the ionized gas in the ICSS FIRGs is so different from that in normal star-forming disk galaxies, we propose that a FIRG be visualized as a galaxy-scale "supergiant" $\mathrm{H}_{\text {II }}$ region: a galaxy whose large-scale interstellar medium is being ionized by UV radiation and possibly mass outflows produced by an intense circumnuclear burst of star formation.

\section{REFERENCES}

Adams, T. F. 1977, Ap. J. Suppl., 33, 19

Adams, T. F., and Weedman, D. 1972, Ap. J., 173, L109.

Armus, L., Heckman, T. M., and Miley, G. K. 1987, A.J., 94, 831 1989, Ap. J., 347, 727

Arp, H. C. 1966, Atlas of Peculiar Galaxies (Pasadena: California Institute of Technology).
Burstein, D., and Heiles, C. 1982, A.J., 87, 1165.

Bushouse, H. A., Lamb, S. A., and Werner, M. W. 1988, Ap. J., 335, 74 (BLW).

Coleman, G. D., Wu, C. C., and Weedman, D. W. 1980, Ap. J. Suppl., 43, 393.

Cottrell, G. A. 1977, M.N.R.A.S., 178, 577.

DePoy, D. L. 1987, Ph.D. thesis, University of Hawaii.

Duric, N., and Seaquist, E. R. 1986, Canadian J. Phys., 64, 531. 
Ford, H. C., Dahari, O., Jacoby, G. H., Crane, P. C., and Ciardullo, R. 1986, Ap. J. (Letters), 311, L7.

Friedman, S. D., Cohen, R. D., Jones, B., Smith, H. E., and Stein, W. A. 1987, A.J., 94, 1480 .

Gehrz, R. D., Sramek, R. A., and Weedman, D. W. 1983, Ap. J., 267, 551

in Gottesman, S. T., and Weliachew, L. 1977, Ap. J., 211, 47.

'- Heckman, T. M., Armus, L., and Miley, G. K. 1987, A.J., 93, 276

Hunter, D. A., and Gallagher, J. S. 1986, Pub. A.S.P., 98, 5 .

Hunter, D. A., Gillett, F. C., Gallagher, J. S., Rice, W. L., and Low, F. J. 1986, Ap. J., 303, 171 .

Hutchings, J. B., Neff, S. G., and van Gorkom, J. H. 1988, A.J., 96, 1227.

Joy, M., Lester, D. F., Harvey, P. M., Telesco, C. M., Decher, R., Rickard, L. J, and Bushouse, H. 1989, Ap. J., 339, 100

Keel, W. C., Kennicutt, R. C., Hummel, E., and van der Hulst, J. M. 1985, A.J., 90, 708 .

Kennicutt, R. C. 1983, Ap. J., 272, 54

- 1984, Ap. J., 287, 116. 1988 , Ap. J, 334, 144

Kennicutt, R. C., and Kent, S. M. 1983, A.J., 88, 1094 (KK).

Kennicutt, R. C., Keel, W. C., van der Hulst, J. M., Hummel, E., and Roettiger, K. A. 1987, A.J.,93, 1011 .

Lonsdale, C. J., Helou, G., Good, J. C., and Rice, W. 1985, Catalogued Galaxies and Quasars Observed in the IRAS Survey (Pasadena: JPL).
McCarthy, P. J., Heckman, T. M., and van Bruegel, W. 1987, A.J., 93, 264.

Markarian, B. E. 1969, Astrofizika, 5, 581

Miller, G. E., and Scalo, J. M. 1979, Ap. J. Suppl., 41, 513

Nilson, P. 1973, Uppsala General Catalogue of Galaxies (Stockholm: Almquist and Wiksell).

Oke, J. B. 1974, Ap. J. Suppl., $27,21$.

Persson, C. J. L., and Helou, G. 1987, Ap. J., 314, 513

Petrosian, A. R. 1980, Astrofizika, 16, 360.

Rieke, G. H., Lebofsky, M. J., Thompson, R. I., Low, F. J., and Tokunaga, A. T. 1980, Ap. J., 238, 24.

Rowan-Robinson, M., and Crawford, J. 1989, M.N.R.A.S., 238, 523.

Sanders, D. B., Soifer, B. J., Elias, J. H., Madore, B. F., Matthews, K., Neugebauer, G., and Scoville, N. Z. 1988, Ap. J., 325, 74

Sanders, D. B., Young J. S., Scoville, N. Z., Soifer, B. T., and Danielson, G. E. 1987, Ap. J. (Letters), 312, L5.

Savage, B. D., and Mathis, J. S. 1979, Ann. Rev. Astr. Ap., 17, 73.

Schecter, P. 1976, Ap. J., 203, 297.

Telesco, C. M., Decher, R, and Gatley, I. 1985, Ap. J. 299, 896

Toomre, A., and Toomre, J. 1972, Ap. J., 178, 623.

Wehinger, P. A., and Wyckoff, S. 1977, M.N.R.A.S., 181, 211

Lee Armus and Timothy M. Heckman: Department of Physics and Astronomy, The Johns Hopkins University, Baltimore, MD 21218

GeORge K. Miley: Lieden Observatory, Niels Bohrweg 2, 2300 RA Leiden, The Netherlands 
PLATE 7

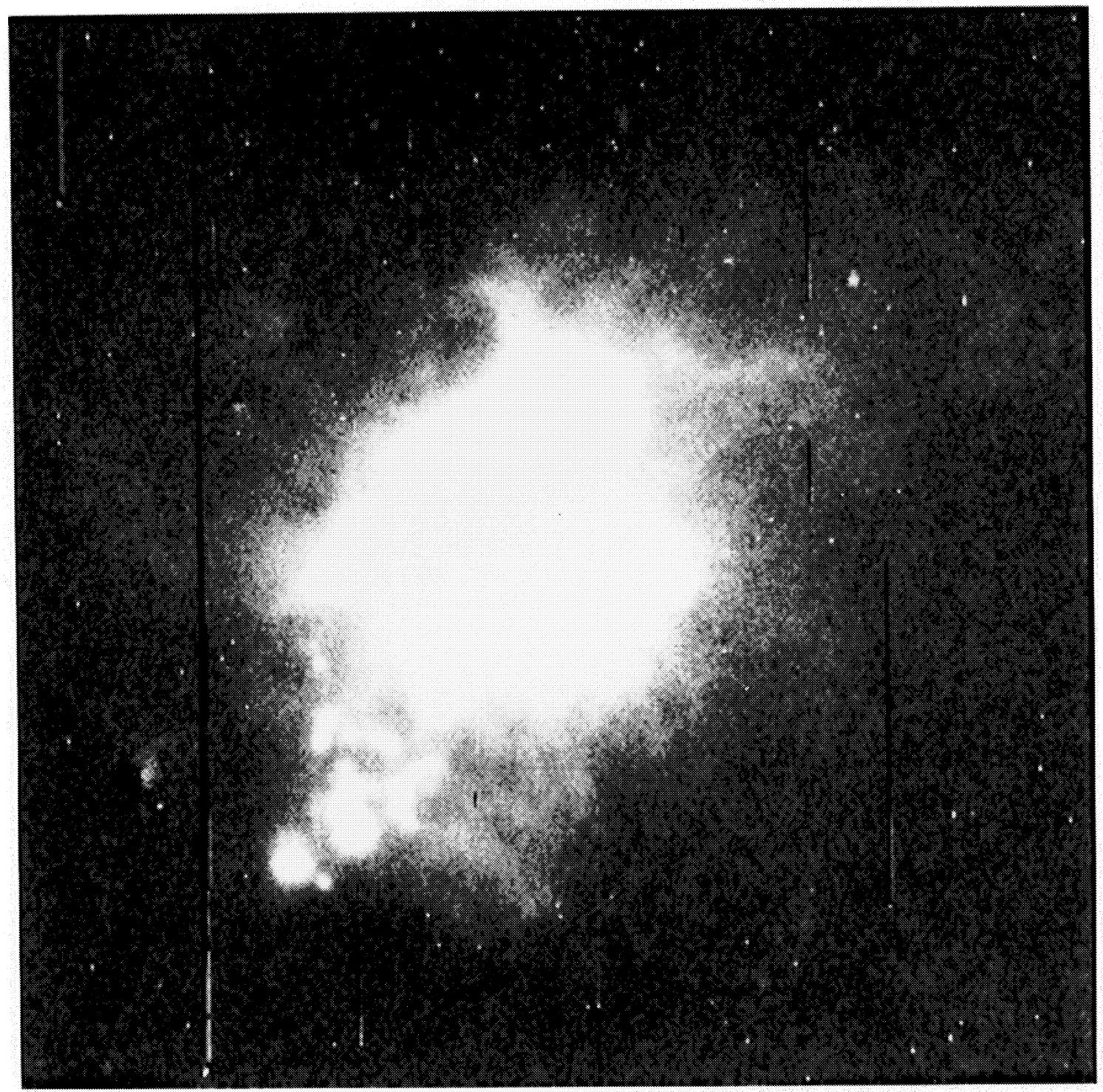

FIG. $8 a$

Fig. 8. - Gray-scale $\mathrm{H} \alpha+[\mathrm{N}$ II] images of (a) NGC 3690, (b) NGC 4194, (c) NGC 660, (d) NGC 3079, (e) Mrk 266, and $(f)$ Mrk 266. In all cases east is toward the top, and north is toward the left. See text for details.

Armus, Heckman, and Miley (see 364, 486) 


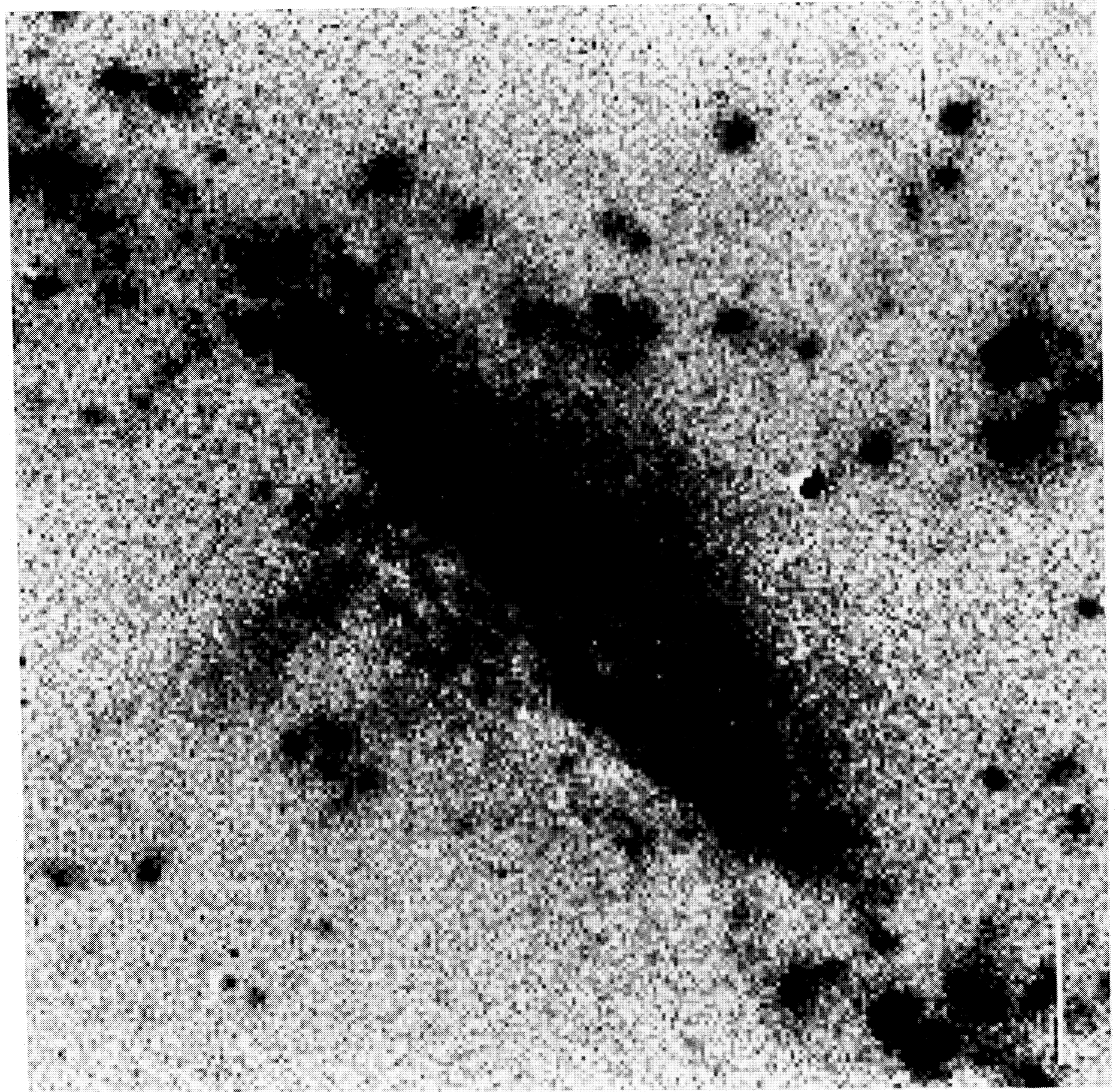

Fig. $8 c$ 


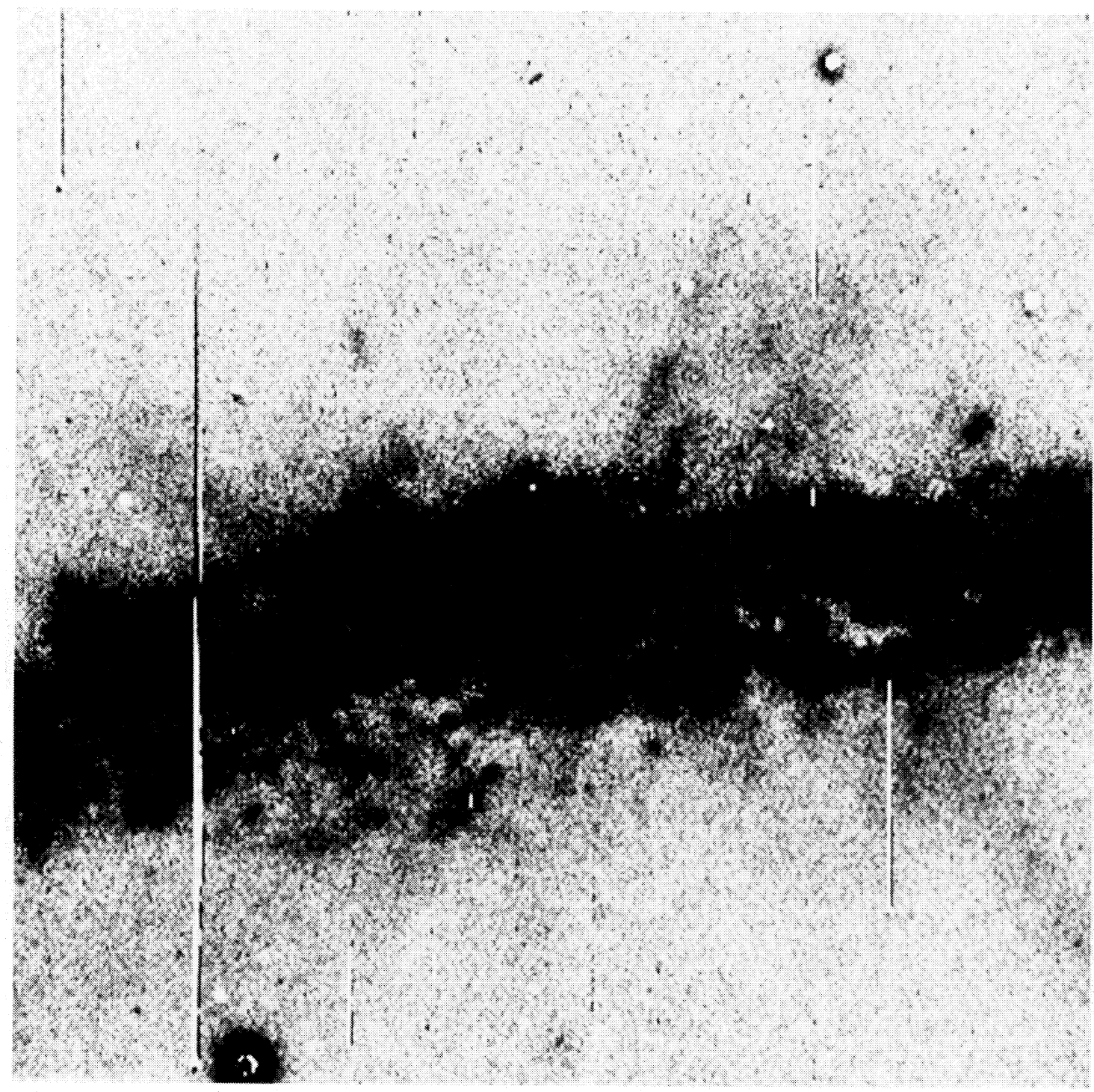

FIG. $8 d$

Armus, Heckman, AND Miley (see 364, 487) 
*
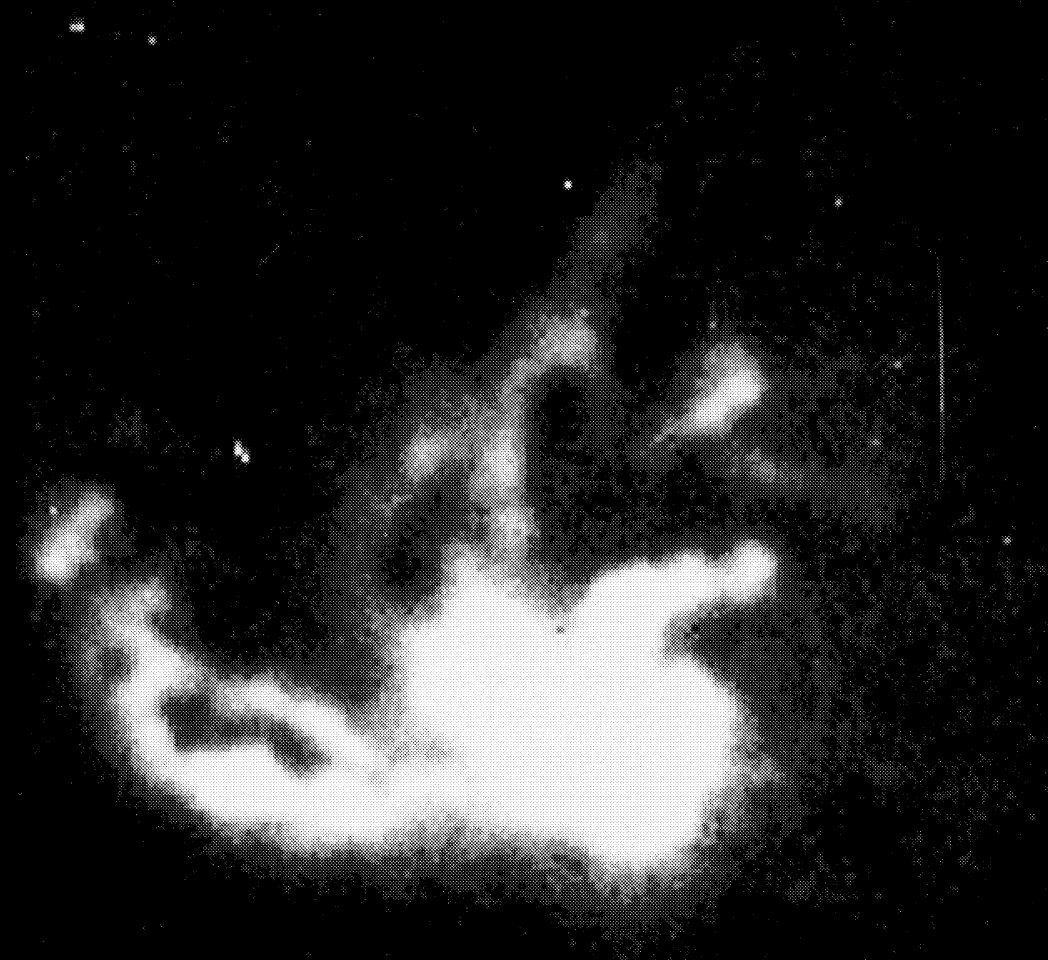


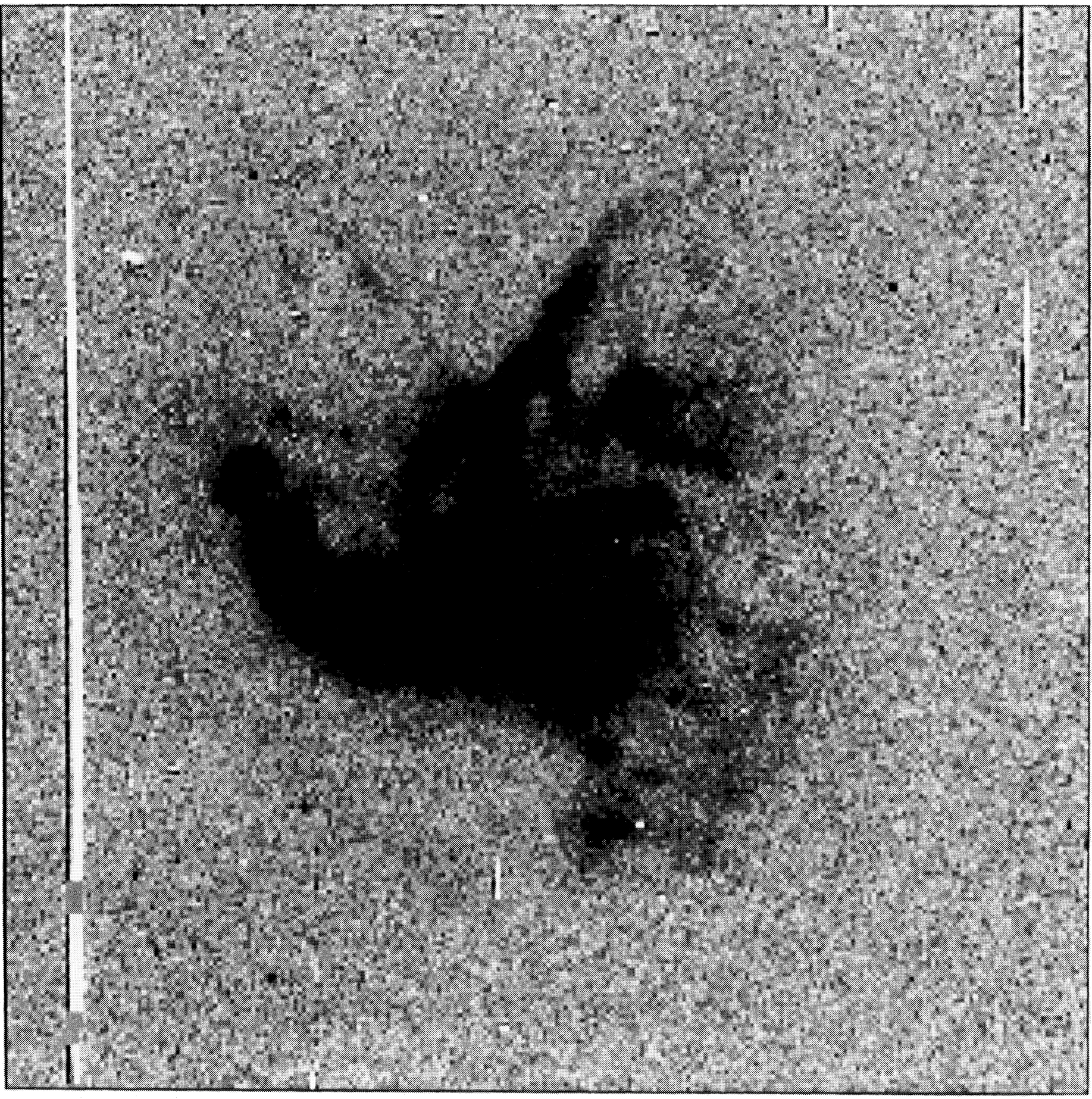

FIG. $8 f$

ARmus, Heckman, AND Miley (see 364, 488) 